\title{
Weighted Sum Synchronization of Memristive Coupled Neural Networks ${ }^{\text {th }}$
}

\author{
Chao Zhou ${ }^{\mathrm{a}}$, Chunhua Wang ${ }^{\mathrm{a}, *}$, Yichuang Sun ${ }^{\mathrm{b}}$, Wei Yao ${ }^{\mathrm{a}}$ \\ ${ }^{a}$ College of Information Science and Engineering, Hunan University, Changsha, 410082, \\ China \\ ${ }^{b}$ School of Engineering and Technology, University of Hertfordshire, Hatfield AL10 9AB, \\ U.K.
}

\begin{abstract}
It is well known that weighted sum of node states plays an essential role in function implementation of neural networks. Therefore, this paper proposes a new weighted sum synchronization model for memristive neural networks. Unlike the existing synchronization models of memristive neural networks which control each network node to reach synchronization, the proposed model treats the networks as dynamic entireties by weighted sum of node states and makes the entireties instead of each node reach expected synchronization. In this paper, weighted sum complete synchronization and quasi-synchronization are both investigated by designing feedback controller and aperiodically intermittent controller, respectively. Meanwhile, a flexible control scheme is designed for the proposed model by utilizing some switching parameters and can improve anti-interference ability of control system. By applying Lyapunov method and some differential inequalities, some effective criteria are derived to ensure the synchronizations of memristive neural networks. Moreover, the error level of the quasi-synchronization is given. Finally, numerical simulation examples are used to certify the effectiveness of the derived results.
\end{abstract}

Key words: Feedback control, intermittent control, Lyapunov function, memristive coupled neural networks, weighted sum synchronization.

\section{Introduction}

Before memristor was discovered, there are three elementary circuit elements: resistor, capacitor and inductor. In 1971, Chua predicted memristor and proved that any combination of the other three circuit elements could not 5 obtain memristor behavior. In 2008, Hewlett-Packard Company succeeded in

\footnotetext{
This work is supported by the National Natural Science Foundation of China (61571185) and the Open Fund Project of Key Laboratory in Hunan Universities (No. 18K010).

* Corresponding author

Email addresses: wch1227164@hnu.edu.cn (Chunhua Wang )
} 
fabricating practical memristor devices, see [1, 2]. Compared to conventional resistor, memristor has better peculiarities such as large density, low power consumption and non-volatile memory. Because of these excellent features, memristor has been successfully applied in different scientific and engineering fields 10 3 3, one of which is to establish memristive neural networks. Different from general nonlinear dynamic systems [8 10, memristive neural network is a kind of state-dependent nonlinear switching system. Therefore, many researchers have studied the dynamic behaviors of memristive neural networks [11 17 .

As a significant collective dynamical behavior, synchronization has drawn much attention owing to its applications in associative memory, information encryption and secure communication [18, 20. Until now, many different kinds of synchronization models have been observed and presented such as global synchronization [21, finite-time synchronization [22, 23, complete synchronization 24 and output synchronization [25. In neural networks, synchronous behavior has a vital role in some functions such as information expression and pattern recognition [26. Thus, plenty of studies on synchronization of memristive neu-

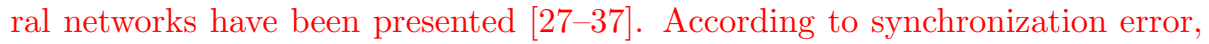
these researches can be divided into two categories: complete synchronization and quasi-synchronization. Complete synchronization whose eventual error is 25 zero means that systems will reach expected synchronization trajectory perfectly. This synchronization is widely studied because it can completely eliminate synchronization error. But in a real-world environment, there exist some interference factors such as outside perturbation and parameter mismatch 38, 39. In these undesirable situations, synchronization error may not be controlled to so zero and then quasi-synchronization is in the spotlight whose final error is nonzero but within an acceptable bound for practical applications. Up to now, many efforts have been devoted to study quasi-synchronization of memristive neural networks 34 37. For example, in [36, Xin et al. investigated quasisynchronization of a kind of chaotic memristive neural networks by treating

35 memristive neural networks as the neural networks with uncertain continuous parameters. In [37, Fan et al. employed some intermittent controllers to tackle quasi-synchronization issue of memristive neural networks with time delay by combining interval matrix algorithm and matrix measure method. As we known, sometimes systems can not be synchronized only by themselves. Thus 40 some control strategies are put forward to drive systems to synchronize, such as sliding-mode control 20, impulsive control 27, adaptive control 40, and aperiodically intermittent control [37, 41. As a discontinuous control strategy, aperiodically intermittent control is practical and economical for engineering applications since it has a unfixed control interval and can reduce information 45 communication effectively. Thus in this paper, this control approach is employed to study the proposed synchronization model.

As introduced above, various synchronization models have been proposed for memristive neural networks. But actually, these synchronization models, according to model structure, can almost be classified as a class of synchronization ${ }_{50}$ pattern: node-to-node synchronization, that is, each node in response system synchronizes with the corresponding node in drive system under controller, as 


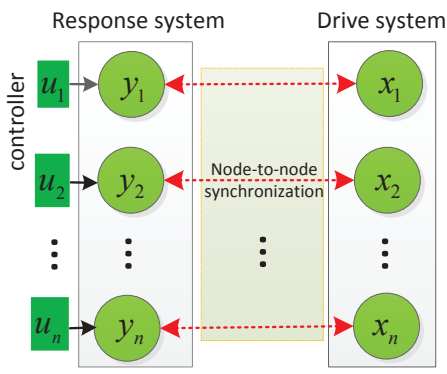

Figure 1: The node-to-node synchronization model of memristive neural networks.

shown in Fig.1. However, this scheme may not be suitable for practical applications because neural networks in reality usually consist of so many neuron nodes and it is difficult or even impossible to control each node to achieve synchronization. Actually in neural networks including artificial and biological neural networks, nodes are always combined by weighted sum form to achieve certain function 42, 43, that is, weighted sum of node states is an essential step in function achievement of neural networks. Thus, it is unnecessary for neural networks to control each node behavior and the control of weighted sum of node 60 states will be more direct and effective for function achievement. Moreover, such a control pattern is economic since the number of controllers can be reduced. Therefore, for neural networks, it is important and profound to propose a synchronization model to study weighted sum of node states. Stimulated by the above analysis, this paper proposes a weighted sum synchronization model for 65 memristive neural networks, as illustrated by Fig. 2 where the synchronization is reached between weighted sums of node states in drive and response systems. The main contributions can be highlighted as follows.

1) A new weighted sum synchronization model is proposed for memristive neural networks (also for neural networks). By designing two different con70 trollers, this paper investigates weighted sum complete synchronization as well as quasi-synchronization. By utilizing Lyapunov function and differential inequalities, the sufficient conditions of two synchronizations are obtained in the paper. Moreover, an explicit expression of the error level is also derived for the quasi-synchronization.

75 2) A flexible control scheme is designed for the proposed model by using some time-varying switching parameters. Changing these parameters, the scheme can adjust controller in real time and thus can deal with some sudden control problems in synchronization process. Therefore, compared with the existing controllers which are fixed and unadjustable in synchronization process, our controller based on this scheme will be more flexible and have a better antiinterference ability.

The remainder of this paper is organized as follows. Section 2 describes model of memristive neural networks and some preliminaries. Section 3 develops some new criteria for weighted sum synchronization of memristive neural 


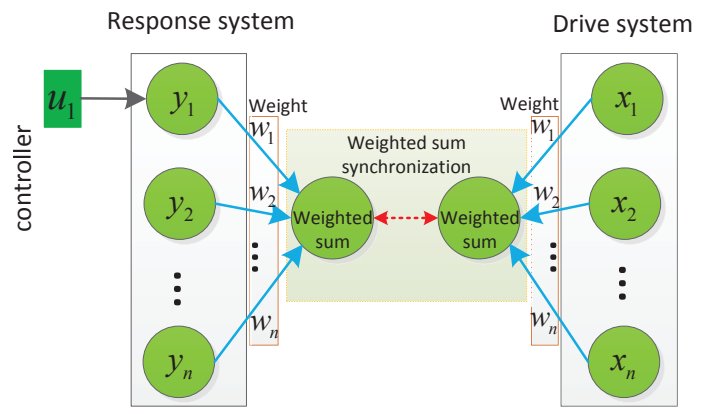

Figure 2: A new weighted sum synchronization model for memristive neural networks.

85

networks. Section 4 gives numerical simulation to demonstrate the effectiveness of proposed results. Finally, some conclusions are presented in Section 5.

Notation: $\overline{c o}\left[\xi^{*}, \xi^{* *}\right]$ denotes the convex hull. For a matrix $A, \lambda_{\max }(A)$ and $\lambda_{\min }(A)$ denote the largest and smallest eigenvalues of $A$, respectively, and $A^{T}$ represents its transposition. $I$ denotes the identity matrix of compatible dimen-

90 sion. For a $\mathrm{n}$-dimension vector $x=\left(x_{1}, \ldots, x_{n}\right),\|x\|$ represents the Euclidian vector norm. For a positive real number $\tau, C([-\tau, 0], \mathbb{R})$ is the family of function $G$ from $[-\tau, 0]$ to $\mathbb{R}$.

\section{Model and preliminaries}

In this paper, based on the early works [21, 22, 30], we consider the following memristive coupled neural networks whose dynamics can be described by state equation:

$$
\begin{aligned}
\dot{x}_{\ell}(t)= & -s_{\ell}\left(x_{\ell}(t)\right) x_{\ell}(t)+\sum_{z=1}^{n} \varphi_{\ell z}\left(x_{\ell}(t)\right) f_{z}\left(x_{z}(t)\right) \\
& +\sum_{z=1}^{n} \phi_{\ell z}\left(x_{\ell}(t)\right) g_{z}\left(x_{z}(t-\varepsilon(t))\right)+I_{\ell}, \ell=1,2, \ldots, n
\end{aligned}
$$

where $f_{z}(\cdot)$ and $g_{z}(\cdot)$ are activation functions, $I_{\ell}$ is the external input, $\varepsilon(t)$ represents time-varying transmission delay, $s_{\ell}\left(x_{\ell}(t)\right), \varphi_{\ell z}\left(x_{\ell}(t)\right)$ and $\phi_{\ell z}\left(x_{\ell}(t)\right)$ are state-dependent memristive coefficients which make the system (1) different from the traditional neural networks [4, 45, whose coefficients are timedependent or some constants. According to the general mathematical model of 
memristor 46, these coefficients can be described as follows:

$$
\begin{array}{r}
s_{\ell}\left(x_{\ell}(t)\right)=\left\{\begin{array}{l}
\overleftarrow{s}_{\ell},\left|x_{\ell}(t)\right| \leq T_{\ell} \\
\vec{s}_{\ell},\left|x_{\ell}(t)\right|>T_{\ell}
\end{array}\right. \\
\varphi_{\ell z}\left(x_{\ell}(t)\right)=\left\{\begin{array}{l}
\overleftarrow{\varphi}_{\ell z},\left|x_{\ell}(t)\right| \leq T_{\ell} \\
\vec{\varphi}_{\ell z},\left|x_{\ell}(t)\right|>T_{\ell}
\end{array}\right. \\
\phi_{\ell z}\left(x_{\ell}(t)\right)=\left\{\begin{array}{l}
\overleftarrow{\phi}_{\ell z},\left|x_{\ell}(t)\right| \leq T_{\ell} \\
\vec{\phi}_{\ell z},\left|x_{\ell}(t)\right|>T_{\ell} .
\end{array}\right.
\end{array}
$$

where switching jumps $T_{\ell}>0, \overleftarrow{s}_{\ell}>0, \vec{s}_{\ell}>0, \overleftarrow{\varphi}_{\ell z}, \vec{\varphi}_{\ell z}, \overleftarrow{\phi}_{\ell z}$ and $\vec{\phi}_{\ell z}, \ell, z=$ $1, \ldots, n$, are all constant numbers.

In the following, we introduce two assumptions which are needed for our study.

Assumption $\left(H_{1}\right)$ : The nonlinear functions $f_{\ell}(\cdot)$ and $g_{\ell}(\cdot)(\ell=1, \ldots, n)$ satisfy

$$
\begin{aligned}
\left|f_{\ell}\left(h_{1}(t)\right)-f_{\ell}\left(h_{2}(t)\right)\right| & \leq a_{\ell}\left|h_{1}(t)-h_{2}(t)\right|, \\
\left|f_{\ell}(.)\right| & \leq l_{1}, \\
\left|g_{\ell}(.)\right| & \leq l_{2},
\end{aligned}
$$

where $h_{1}(t), h_{2}(t) \in \mathbb{R}, a_{\ell}>0, l_{1}>0$, and $l_{2}>0$ are some known constants.

Assumption $\left(H_{2}\right)$ : The time delay $\varepsilon(t)$ satisfies $0<\varepsilon(t) \leq \mu$, where $\mu>0$.

From (2), it is easy to see that $s_{\ell}\left(x_{\ell}(t)\right), \varphi_{\ell z}\left(x_{\ell}(t)\right)$ and $\phi_{\ell z}\left(x_{\ell}(t)\right)$ are discontinuous. Therefore, the solutions of (1) are handled in Filippov's sense. Now, we give the following definition.

Definition 1 (Filippov Regularization [47): The Filippov set-valued map of $f(t, x)$ at $x \in \mathbb{R}^{n}$ is defined as follows:

$$
F(t, x)=\bigcap_{\delta>0} \bigcap_{\mu(N)=0} \overline{\mathrm{co}}[f(B(x, \delta) \backslash N)]
$$

where $F(t, x)=\bigcap_{\delta>0} \bigcap_{\mu(N)=0} \overline{\operatorname{co}}[f(B(x, \delta) \backslash N)]$, and $\mu(N)$ is the Lebesgue measure of set $N . \overline{\mathrm{co}}[E]$ denotes the closure of the convex hull of the set $E$.

Then, according to Definition 1 and by applying the theory of differential inclusion, we can get the memristive neural networks:

$$
\begin{aligned}
\dot{x}_{\ell} \in & -\overline{\mathrm{co}}\left(s_{\ell}^{*}, s_{\ell}^{* *}\right) x_{\ell}(t)+\sum_{z=1}^{n} \overline{\mathrm{co}}\left(\varphi_{\ell z}^{*}, \varphi_{\ell z}^{* *}\right) f_{z}\left(x_{z}(t)\right) \\
& +\sum_{z=1}^{n} \overline{\operatorname{co}}\left(\phi_{\ell z}^{*}, \phi_{\ell z}^{* *}\right) g_{z}\left(x_{z}(t-\varepsilon(t))\right)+I_{\ell}, \ell=1,2, \ldots, n
\end{aligned}
$$

where $s_{\ell}^{*}=\min \left\{\overleftarrow{s}_{\ell}, \vec{s}_{\ell}\right\}, s_{\ell}^{* *}=\max \left\{\overleftarrow{s}_{\ell}, \vec{s}_{\ell}\right\}, \varphi_{\ell z}^{*}=\min \left\{\overleftarrow{\varphi}_{\ell z}, \vec{\varphi}_{\ell z}\right\}, \varphi_{\ell z}^{* *}=$ $\max \left\{\overleftarrow{\varphi}_{\ell z}, \vec{\varphi}_{\ell z}\right\}, \phi_{\ell z}^{*}=\min \left\{\overleftarrow{\phi}_{\ell z}, \vec{\phi}_{\ell z}\right\}, \phi_{\ell z}^{* *}=\max \left\{\overleftarrow{\phi}_{\ell z}, \vec{\phi}_{\ell z}\right\}$, and 


$$
\begin{gathered}
\overline{\operatorname{co}}\left(s_{\ell}^{*}, s_{\ell}^{* *}\right)=\left\{\begin{array}{l}
\overleftarrow{s}_{\ell},\left|x_{\ell}(t)\right|<T_{\ell} \\
{\left[s_{\ell}^{*}, s_{\ell}^{* *}\right],\left|x_{\ell}(t)\right|=T_{\ell}} \\
\vec{s}_{\ell},\left|x_{\ell}(t)\right|>T_{\ell}
\end{array}\right. \\
\overline{\operatorname{co}}\left(\varphi_{\ell z}^{*}, \varphi_{\ell z}^{* *}\right)=\left\{\begin{array}{l}
\overleftarrow{\varphi}_{\ell z},\left|x_{\ell}(t)\right|<T_{\ell} \\
{\left[\varphi_{\ell z}^{*}, \varphi_{\ell z}^{* *}\right],\left|x_{\ell}(t)\right|=T_{\ell}} \\
\vec{\varphi}_{\ell z},\left|x_{\ell}(t)\right|>T_{\ell}
\end{array}\right. \\
\overline{\operatorname{co}}\left(\phi_{\ell z}^{*}, \phi_{\ell z}^{* *}\right)=\left\{\begin{array}{l}
\overleftarrow{\phi}_{\ell z},\left|x_{\ell}(t)\right|<T_{\ell} \\
{\left[\phi_{\ell z}^{*}, \phi_{\ell z}^{* *}\right],\left|x_{\ell}(t)\right|=T_{\ell}} \\
\vec{\phi}_{\ell z},\left|x_{\ell}(t)\right|>T_{\ell} .
\end{array}\right.
\end{gathered}
$$

In this paper, the corresponding response system with control is considered as:

$$
\begin{aligned}
\dot{y}_{\ell}(t)= & -s_{\ell}\left(y_{\ell}(t)\right) y_{\ell}(t)+\sum_{z=1}^{n} \varphi_{\ell z}\left(y_{\ell}(t)\right) f_{z}\left(y_{z}(t)\right) \\
& +\sum_{z=1}^{n} \phi_{\ell z}\left(y_{\ell}(t)\right) g_{z}\left(y_{z}(t-\varepsilon(t))\right)+I_{\ell}+u_{\ell}, \ell=1,2, \ldots, n
\end{aligned}
$$

where

$$
\begin{aligned}
& s_{\ell}\left(y_{\ell}(t)\right)=\left\{\begin{array}{l}
\overleftarrow{s}_{\ell},\left|y_{\ell}(t)\right| \leq T_{\ell} \\
\vec{s}_{\ell},\left|y_{\ell}(t)\right|>T_{\ell}
\end{array}\right. \\
& \varphi_{\ell z}\left(y_{\ell}(t)\right)=\left\{\begin{array}{l}
\overleftarrow{\varphi}_{\ell z},\left|y_{\ell}(t)\right| \leq T_{\ell} \\
\vec{\varphi}_{\ell z},\left|y_{\ell}(t)\right|>T_{\ell}
\end{array}\right. \\
& \phi_{\ell z}\left(y_{\ell}(t)\right)=\left\{\begin{array}{l}
\overleftarrow{\phi}_{\ell z},\left|y_{\ell}(t)\right| \leq T_{\ell} \\
\vec{\phi}_{\ell z},\left|y_{\ell}(t)\right|>T_{\ell} .
\end{array}\right.
\end{aligned}
$$

$u_{\ell}(t)$ is the controller to be designed.

Similar to (3), the response system can be written as follows:

$$
\begin{aligned}
\dot{y}_{\ell} \in & -\overline{\mathrm{co}}\left(s_{\ell}^{*}, s_{\ell}^{* *}\right) y_{\ell}(t)+\sum_{z=1}^{n} \overline{\mathrm{co}}\left(\varphi_{\ell z}^{*}, \varphi_{\ell z}^{* *}\right) f_{z}\left(y_{z}(t)\right) \\
& +\sum_{z=1}^{n} \overline{\mathrm{co}}\left(\phi_{\ell z}^{*}, \phi_{\ell z}^{* *}\right) g_{z}\left(y_{z}(t-\varepsilon(t))\right)+I_{\ell}+u_{\ell}, \ell=1,2, \ldots, n
\end{aligned}
$$

where

$$
\begin{aligned}
\overline{\operatorname{co}}\left(s_{\ell}^{*}, s_{\ell}^{* *}\right)=\left\{\begin{array}{l}
\overleftarrow{s}_{\ell},\left|y_{\ell}(t)\right|<T_{\ell} \\
{\left[s_{\ell}^{*}, s_{\ell}^{* *}\right],\left|y_{\ell}(t)\right|=T_{\ell}} \\
\vec{s}_{\ell},\left|y_{\ell}(t)\right|>T_{\ell}
\end{array}\right. \\
\overline{\operatorname{co}}\left(\varphi_{\ell z}^{*}, \varphi_{\ell z}^{* *}\right)=\left\{\begin{array}{l}
\overleftarrow{\varphi}_{\ell z},\left|y_{\ell}(t)\right|<T_{\ell} \\
{\left[\varphi_{\ell z}^{*}, \varphi_{\ell z}^{* *},\left|y_{\ell}(t)\right|=T_{\ell}\right.} \\
\vec{\varphi}_{\ell z},\left|y_{\ell}(t)\right|>T_{\ell}
\end{array}\right.
\end{aligned}
$$




$$
\overline{\operatorname{co}}\left(\phi_{\ell z}^{*}, \phi_{\ell z}^{* *}\right)=\left\{\begin{array}{l}
\overleftarrow{\phi}_{\ell z},\left|y_{\ell}(t)\right|<T_{\ell} \\
{\left[\phi_{\ell z}^{*}, \phi_{\ell z}^{* *}\right],\left|y_{\ell}(t)\right|=T_{\ell}} \\
\vec{\phi}_{\ell z},\left|y_{\ell}(t)\right|>T_{\ell} .
\end{array}\right.
$$

Now, we give the definitions of weighted sum complete synchronization and quasi-synchronization, and some lemmas which will help to derive the main results of our work.

Definition 2: The weighted sum complete synchronization of drive-response memristive neural networks (1) and (4) is said to be achieved if, by designing suitable controllers $u_{\ell}(t), \ell=1,2, \ldots, n$, there are the nonnegative weights $w_{\ell}, \ell=$ $1,2, \ldots, n$, such that for any initial values $x(t), y(t) \in C([-\mu, 0] ; \mathbb{R})$,

$$
\lim _{t \rightarrow \infty}\left\|\sum_{\ell=1}^{n} w_{\ell} y_{\ell}(t)-\sum_{\ell=1}^{n} w_{\ell} x_{\ell}(t)\right\|=0 .
$$

Definition 3: The weighted sum quasi-synchronization of drive-response memristive neural networks (1) and (4) is said to be achieved if, by designing suitable controllers $u_{\ell}(t), \ell=1,2, \ldots, n$, there are constants $\partial>0, w_{\ell} \geq$ $0, \ell=1,2, \ldots, n$, such that for any initial values $x(t), y(t) \in C([-\mu, 0] ; \mathbb{R})$,

$$
\lim _{t \rightarrow \infty}\left\|\sum_{\ell=1}^{n} w_{\ell} y_{\ell}(t)-\sum_{\ell=1}^{n} w_{\ell} x_{\ell}(t)\right\| \leq \partial .
$$

Remark 1: Recently, output synchronization has been investigated in 25] and [48 51]. In these researches, each network node $x_{i}$ has multiple states $x_{\ell} \in \mathbb{R}^{p}, p>1$, and node output is expressed as $o_{\ell}=b x_{\ell}$, where $b$ is a constant matrix. In these researches, the synchronization is achieved between node outputs. From synchronization form, there is similarity between output synchronization and the proposed synchronization since they both combined node states by using a matrix $b$ or a vector $\left(w_{1}, \ldots, w_{n}\right)$. But in fact, our work is different from these researches. First, output coupling condition and dissipation coupling condition (i.e., the sum of each row of coupling configuration matrix 120 must be zero ), some strong conditions for memristive neural networks, do not need to be satisfied in our work but play a crucial role in the synchronization achievements of 25 and 48 51. Therefore, their obtained results are not suitable for this paper at all. On the other hand, different from general nonlinear networks in 25] and [48 51, memristive neural network, a more complex statedependent switching system, is considered in this paper, which makes our work more challenging and difficult.

Lemma 1 ([52]): For chosen non-negative continuous function $g(t)$, if it satisfies: $\dot{g}(t) \leq-\alpha g(t)+\beta g(t-\varepsilon(t)), t \geq t_{0}$, where $\alpha>\beta>0,0<\varepsilon(t) \leq \mu$.

Then, $g(t) \leq g_{\mu}\left(t_{0}\right) e^{-r\left(t-t_{0}\right)}, t \geq t_{0}$, where $g_{\mu}\left(t_{0}\right)=\sup _{t_{0}-\mu \leq s \leq t_{0}}\{g(s)\}$ and 130 $\quad r>0$ is the unique positive solution of the equation $r-\alpha+\beta e^{r \mu}=0$.

Lemma 2 [37]: For chosen continuous non-negative function $g(t)$, if it satisfies: $\dot{g}(t) \leq-\alpha g(t)+\beta g(t-\varepsilon(t))+\psi, t \geq t_{0}$, where $\alpha>\beta>0, \psi>0$, $0<\varepsilon(t) \leq \mu$. 
Then, $g(t) \leq g_{\mu}\left(t_{0}\right) e^{-\varpi\left(t-t_{0}\right)}+\frac{\psi}{\varpi}, t \geq t_{0}$, where $g_{\mu}\left(t_{0}\right)=\sup _{t_{0}-\mu \leq s \leq t_{0}}\{g(s)\}$,

$\varpi$ is the unique positive solution of the equation $-\varpi=-\alpha+\beta e^{\varpi \mu}$.

Lemma 3 [37]: For chosen continuous function $g(t)$, if it is non-negative and satisfies: $\dot{g}(t) \leq \alpha g(t)+\beta g(t-\varepsilon(t))+\psi, t>t_{0}$, where $\beta, \alpha+\beta$, and $\psi$ are positive scalars, $0<\varepsilon(t) \leq \mu$.

Then, the following inequality holds:

$$
g(t) \leq\left(g_{\mu}\left(t_{0}\right)+\frac{\psi}{\alpha+\beta}\right) e^{(\alpha+\beta)\left(t-t_{0}\right)}-\frac{\psi}{\alpha+\beta}, t \geq t_{0}
$$

where $g_{\mu}\left(t_{0}\right)=\sup _{t_{0}-\mu \leq s \leq t_{0}}\{g(s)\}$.

Lemma 4 [53]: For chosen function $g(t)$, if it is non-negative and satisfies:

$$
\begin{gathered}
g(t) \leq g_{\mu}\left(t_{r}\right) e^{-\varpi\left(t-t_{r}\right)}+\varsigma, t_{r} \leq t<\tau_{r} \\
g(t) \leq\left(g_{\mu}\left(\tau_{r}\right)+\xi\right) e^{\omega\left(t-\tau_{r}\right)}-\xi, \tau_{r} \leq t<\mathrm{t}_{r+1} .
\end{gathered}
$$

Then, the following inequality holds:

$$
g(t) \leq g_{\mu}(0) e^{h} e^{-(h / \sigma) t}+\frac{\rho}{1-e^{-h}}+\varsigma, t>0 .
$$

where $h=h_{1}-h_{2}>0, h_{1}=\varpi(\theta-\mu), h_{2}=\omega(\sigma-\theta), \theta=\inf _{r}\left(\tau_{r}-t_{r}\right)$, $\sigma=\sup _{r}\left(t_{r+1}-t_{r}\right), \rho=(\varsigma+\xi) e^{h_{2}}-\xi$.

Lemma 5: For any vectors $q, p \in \mathbb{R}^{n}$, there exists the following inequality.

$$
2 q^{T} p \leq q^{T} q+p^{T} p .
$$

\section{Main results}

In this section, state feedback control is first considered for weighted sum complete synchronization of the drive-response systems (1) and (4). Then a flexible aperiodically intermittent control scheme is designed to ensure weighted sum quasi-synchronization of the systems. By constructing Lyapunov function and applying some differential inequalities, we will derive the sufficient conditions of two synchronizations in this section.

Define the node error signal as $e_{\ell}(t)=y_{\ell}(t)-x_{\ell}(t), \ell=1,2, \ldots, n$. For convenience of the following proof, let

$W=\left(w_{1}, \ldots, w_{n}\right), e(t)=\left(e_{1}, \ldots, e_{n}\right)^{T}, S=\operatorname{diag}\left(s_{1}\left(x_{1}(t)\right), \ldots, s_{n}\left(x_{n}(t)\right)\right)$, $K=\left(k_{\ell z}(t)\right)_{n \times n}, \vec{S}=\operatorname{diag}\left(\vec{s}_{1}, \ldots, \vec{s}_{n}\right), \overleftarrow{S}=\operatorname{diag}\left(\overleftarrow{s}_{1}, \ldots, \overleftarrow{s}_{n}\right), \vec{\varphi}=\left(\vec{\varphi}_{\ell z}\right)_{n \times n}, \overleftarrow{\varphi}=$ $\left(\overleftarrow{\varphi}_{\ell z}\right)_{n \times n}, \vec{\phi}=\left(\vec{\phi}_{\ell z}\right)_{n \times n}, \overleftarrow{\phi}=\left(\overleftarrow{\phi}_{\ell z}\right)_{n \times n}, U=\left(u_{1}, \ldots, u_{n}\right)^{T}, A=\operatorname{diag}\left(a_{1}, \ldots, a_{n}\right)$ $\Pi_{\ell}^{x}=\left(\overleftarrow{s}_{\ell}-\vec{s}_{\ell}\right) x_{\ell}(t)+\sum_{z=1}^{n}\left(\vec{\varphi}_{\ell z}-\overleftarrow{\varphi}_{\ell z}\right) f_{z}\left(x_{z}(t)\right)+\sum_{z=1}^{n}\left(\vec{\phi}_{\ell z}-\overleftarrow{\phi}_{\ell z}\right) g_{z}\left(x_{z}(t-\varepsilon(t))\right)$, $\Pi_{\ell}^{y}=\left(\vec{s}_{\ell}-\overleftarrow{s}_{\ell}\right) y_{\ell}(t)+\sum_{z=1}^{n}\left(\overleftarrow{\varphi}_{\ell z}-\vec{\varphi}_{\ell z}\right) f_{z}\left(y_{z}(t)\right)+\sum_{z=1}^{n}\left(\overleftarrow{\phi}_{\ell z}-\vec{\phi}_{\ell z}\right) g_{z}\left(y_{z}(t-\varepsilon(t))\right)$ $\Pi_{\ell}=\left|\overleftarrow{s}_{\ell}-\vec{s}_{\ell}\right| T_{\ell}+\sum_{z=1}^{n} a_{z}\left|\overleftarrow{\varphi}_{\ell z}-\vec{\varphi}_{\ell z}\right| T_{z}+\sum_{z=1}^{n}\left|\overleftarrow{\phi}_{\ell z}-\vec{\phi}_{\ell z}\right| l_{2}, \Pi^{y}=\left(\Pi_{1}^{y}, \ldots, \Pi_{n}^{y}\right)^{T}$, $\Pi^{x}=\left(\Pi_{1}^{x}, \ldots, \Pi_{n}^{x}\right)^{T}, \Pi=\left(\Pi_{1}, \ldots, \Pi_{n}\right)^{T}$. 


\subsection{Weighted sum complete synchronization}

For weighted sum complete synchronization, only one state feedback controller is designed to reduce control cost. Without loss of generality, assume the weight of the first node is non-zero and the controller can be designed as follows:

$$
\left\{\begin{aligned}
u_{1}(t)= & \sum_{\ell=1}^{n} \frac{w_{\ell}}{w_{1}}\left[\sum_{z=1}^{n} k_{\ell z}(t)\left(x_{z}(t)-y_{z}(t)\right)\right. \\
& \left.+\eta_{\ell}\left(\operatorname{sign}\left(\sum_{z=1}^{n} w_{z}\left(x_{z}(t)-y_{z}(t)\right)\right)\right)\right], w_{1} \neq 0 \\
u_{\ell}(t)= & 0, \ell=2,3, \ldots, n
\end{aligned}\right.
$$

where $w_{\ell}$ denotes weight parameter, $k_{\ell z}(t)$ and $\eta_{\ell}$ are the control gain parameters, and $\operatorname{sign}(\cdot)$ is the standard sign function.

Theorem 1: By applying Assumptions $\left(H_{1}\right)$ and $\left(H_{2}\right)$, the weighted sum complete synchronization of drive system (1) and response system (4) is achieved under the synchronization controller (5), if for positive constants $q, \overleftarrow{M}_{\ell}, \vec{M}_{\ell}, \overleftarrow{c}_{\ell}$ and $\vec{c}_{\ell},(\ell=1,2)$, control gain $K$ and $\eta_{\ell}$ satisfy condition $\left.C 1\right)$ and condition C2), respectively.

$C 1): W(-S-K)=-q W$, where $q$ satisfies $\alpha=\min \left(q-\vec{c}_{1}-\frac{\vec{c}_{2}}{2}, q-\overleftarrow{c}_{1}-\frac{\overleftarrow{c}_{2}}{2}\right)>$ $\beta=\max \left(\frac{\overleftarrow{c_{2}}}{2}, \frac{\vec{c}_{2}}{2}\right)$.

C2): $\sum_{\ell=1}^{n} w_{\ell} \eta_{\ell}>\gamma=\sum_{\ell=1}^{n} w_{\ell} \Pi_{\ell}+\max \left(\vec{M}_{1}+\vec{M}_{2}, \overleftarrow{M}_{1}+\overleftarrow{M}_{2}\right)$

Proof: Construct a Lyapunov-Krasovskii function as follows:

$$
V(t)=\frac{1}{2}(W e(t))^{T} W e(t) .
$$

Since connection coefficients of memristive neural networks are state-dependent, the proof can be divided into the following four cases.

Case 1: If $\left|x_{\ell}(t)\right| \leq T_{\ell},\left|y_{\ell}(t)\right| \leq T_{\ell}$ at time $t$, we can obtain the error system

$$
\dot{e}_{\ell}(t)=-\overleftarrow{s}_{\ell} e_{\ell}(t)+\sum_{z=1}^{n} \overleftarrow{\varphi}_{\ell z} \tilde{f}_{z}\left(e_{z}(t)\right)+\sum_{z=1}^{n} \overleftarrow{\phi}_{\ell z} \tilde{g}_{z}\left(e_{z}(t-\varepsilon(t))\right)+u_{\ell}
$$

where $\tilde{f}_{z}\left(e_{z}(t)\right)=f_{z}\left(y_{z}(t)\right)-f_{z}\left(x_{z}(t)\right)$ and $\tilde{g}_{z}\left(e_{z}(t-\varepsilon(t))\right)=g_{z}\left(y_{z}(t-\varepsilon(t))\right)-$ $g_{z}\left(x_{z}(t-\varepsilon(t))\right)$.

By Assumption $\left(H_{1}\right)$, we can get

$$
\begin{aligned}
(W e(t))^{T} W \overleftarrow{\varphi} \tilde{f}(e(t)) & =(W e(t))^{T} \sum_{z=1}^{n} \tilde{f}_{z}\left(e_{z}(t)\right) \sum_{\ell=1}^{n} w_{\ell} \overleftarrow{\varphi}_{\ell z} \\
& \leq|W e(t)| \sum_{z=1}^{n}\left|\tilde{f}_{z}\left(e_{z}(t)\right)\right|\left|\sum_{\ell=1}^{n} w_{\ell} \overleftarrow{\varphi}_{\ell z}\right| \\
& \leq 2 l_{1}|W e(t)| \sum_{z=1}^{n}\left|\sum_{\ell=1}^{n} w_{\ell} \overleftarrow{\varphi}_{\ell z}\right|
\end{aligned}
$$


The $2 l_{1} \sum_{z=1}^{n}\left|\sum_{\ell=1}^{n} w_{\ell} \overleftarrow{\varphi}_{\ell z}\right|$ is a bounded constant, which means that there are positive constants $\overleftarrow{M}_{1}$ and $\overleftarrow{c}_{1}$, such that

$$
2 l_{1} \sum_{z=1}^{n}\left|\sum_{\ell=1}^{n} w_{\ell} \overleftarrow{\varphi}_{\ell z}\right| \leq \overleftarrow{M}_{1}+\overleftarrow{c}_{1}|W e(t)|
$$

Thus

$$
(W e(t))^{T} W \overleftarrow{\varphi} \tilde{f}(e(t)) \leq \overleftarrow{M}_{1}\left|(W e(t))^{T}\right|+\overleftarrow{c}_{1}(W e(t))^{T} W e(t)
$$

Similarly

$$
\begin{aligned}
\left.(W e(t))^{T} W \overleftarrow{\phi} \tilde{g}(e(t-\varepsilon(t)))\right) & \leq|W e(t)| \sum_{j=1}^{n} 2 l_{2}\left|\sum_{\ell=1}^{n} w_{\ell} \overleftarrow{\phi}_{\ell z}\right| \\
& \leq|W e(t)|\left(\overleftarrow{M}_{2}+\overleftarrow{c}_{2}|W e(t-\varepsilon(t))|\right) \\
& =\overleftarrow{M}_{2}\left|(W e(t))^{T}\right|+\overleftarrow{c}_{2}\left|(W e(t))^{T}\right||W e(t-\varepsilon(t))|
\end{aligned}
$$

According to Lemma 5, we can obtain

$$
\begin{aligned}
\left.(W e(t))^{T} W \overleftarrow{\phi} \tilde{g}(e(t-\varepsilon(t)))\right) & \leq \overleftarrow{M}_{2}\left|(W e(t))^{T}\right|+\frac{\overleftarrow{c}_{2}}{2}(W e(t))^{T} W e(t) \\
& +\frac{\overleftarrow{c}_{2}}{2}(W e(t-\varepsilon(t)))^{T} W e(t-\varepsilon(t)) .
\end{aligned}
$$

Note that $W U=-W K e(t)-\sum_{i=1}^{n} w_{i} \eta_{i} \operatorname{sign}(W e(t))$.

Based on the above results, we can derive the time derivative of $V(t)$ as follows

$$
\begin{aligned}
D^{+} V(t)= & (W e(t))^{T} W(-\overleftarrow{S} e(t)+\overleftarrow{\varphi} \tilde{f}(e(t))+\overleftarrow{\phi} \tilde{g}(e(t-\varepsilon(t)))+U) \\
\leq & (W e(t))^{T}\left(-W \overleftarrow{S} e(t)+\overleftarrow{c}_{1} W e(t)+\frac{\overleftarrow{c}_{2}}{2} W e(t)-W K e(t)\right) \\
& +\overleftarrow{M}_{1}\left|(W e(t))^{T}\right|+\overleftarrow{M}_{2}\left|(W e(t))^{T}\right| \\
& +\frac{\overleftarrow{c}_{2}}{2}(W e(t-\varepsilon(t)))^{T} W e(t-\varepsilon(t))-\sum_{\ell=1}^{n} w_{\ell} \eta_{\ell}\left|(W e(t))^{T}\right| \\
= & \left(\overleftarrow{c}_{1}+\frac{\overleftarrow{c}_{2}}{2}\right)(W e(t))^{T} W e(t)+(W e(t))^{T} W(-\overleftarrow{S}-K) e(t) \\
& +\frac{\overleftarrow{c}_{2}}{2}(W e(t-\varepsilon(t)))^{T} W e(t-\varepsilon(t)) \\
& -\left(\sum_{\ell=1}^{n} w_{\ell} \eta_{\ell}-\overleftarrow{M}_{1}-\overleftarrow{M}_{2}\right)\left|(W e(t))^{T}\right|
\end{aligned}
$$


Then, from the conditions C1) and C2), we obtain

$$
\begin{aligned}
D^{+} V(t) & \leq-\left(q-\overleftarrow{c}_{1}-\frac{\overleftarrow{c}_{2}}{2}\right)(W e(t))^{T} W e(t) \\
& +\frac{\overleftarrow{c}_{2}}{2}(W e(t-\varepsilon(t)))^{T} W e(t-\varepsilon(t)) \\
& \leq-\alpha V(t)+\beta V(t-\varepsilon(t)) .
\end{aligned}
$$

Case 2: If $\left|x_{\ell}(t)\right|>T_{\ell}\left|y_{\ell}(t)\right|>T_{\ell}$ at time $t$, we have the error system

$$
\dot{e}_{\ell}(t)=-\vec{s}_{\ell} e_{\ell}(t)+\sum_{z=1}^{n} \vec{\varphi}_{\ell z} \tilde{f}_{z}\left(e_{z}(t)\right)+\sum_{z=1}^{n} \vec{\phi}_{\ell z} \tilde{g}_{z}\left(e_{z}(t-\varepsilon(t))\right)+u_{\ell}
$$

Analogously, there are positive constants $\vec{M}_{\ell}$ and $\vec{c}_{\ell}(\ell=1,2)$, such that

$$
\begin{aligned}
D^{+} V(t) & \leq\left(\vec{c}_{1}+\frac{\vec{c}_{2}}{2}\right)(W e(t))^{T} W e(t)+(W e(t))^{T} W(-\vec{S}-K) e(t) \\
& +\frac{\vec{c}_{2}}{2}(W e(t-\varepsilon(t)))^{T} W e(t-\varepsilon(t)) \\
& -\left(\sum_{\ell=1}^{n} w_{\ell} \eta_{\ell}-\vec{M}_{1}-\vec{M}_{2}\right)\left|(W e(t))^{T}\right| \\
& =\left(-q+\vec{c}_{1}+\frac{\vec{c}_{2}}{2}\right)(W e(t))^{T} W e(t) \\
& +\frac{\vec{c}_{2}}{2}(W e(t-\varepsilon(t)))^{T} W e(t-\varepsilon(t)) \\
& \leq-\alpha V(t)+\beta V(t-\varepsilon(t)) .
\end{aligned}
$$

Case 3: If $\left|x_{\ell}(t)\right|>T_{\ell},\left|y_{\ell}(t)\right| \leq T_{\ell}$ at time $t$, we rewrite the error system as $\dot{e}_{\ell}(t)=-\vec{s}_{\ell} e_{\ell}(t)+\sum_{z=1}^{n} \vec{\varphi}_{\ell z} \tilde{f}_{z}\left(e_{z}(t)\right)+\sum_{z=1}^{n} \vec{\phi}_{\ell z} \tilde{g}_{z}\left(e_{z}(t-\varepsilon(t))\right)+\Pi_{\ell}^{y}+u_{\ell}$.

Noting that $\left|\Pi_{\ell}^{y}\right| \leq \Pi_{\ell}$, we can calculate the derivative of $V(t)$ as

$$
\begin{aligned}
D^{+} V(t) & \leq\left(\vec{c}_{1}+\frac{\overrightarrow{c_{2}}}{2}\right)(W e(t))^{T} W e(t)+(W e(t))^{T} W(-\vec{S}-K) e(t) \\
& +\frac{\vec{c}_{2}}{2}(W e(t-\varepsilon(t)))^{T} W e(t-\varepsilon(t)) \\
& -\left(\sum_{\ell=1}^{n} w_{\ell}\left(\eta_{\ell}-\Pi_{\ell}\right)-\vec{M}_{1}-\vec{M}_{2}\right)\left|(W e(t))^{T}\right|
\end{aligned}
$$

From conditions C1) and C2), we can obtain

$$
\begin{aligned}
D^{+} V(t) & \leq\left(-q+\vec{c}_{1}+\frac{\vec{c}_{2}}{2}\right)(W e(t))^{T} W e(t) \\
& +\frac{\vec{c}_{2}}{2}(W e(t-\varepsilon(t)))^{T} W e(t-\varepsilon(t)) \\
& \leq-\alpha V(t)+\beta V(t-\varepsilon(t)) .
\end{aligned}
$$


Case 4: If $\left|x_{\ell}(t)\right| \leq T_{\ell},\left|y_{\ell}(t)\right|>T_{\ell}$ at time $t$, we obtain the corresponding error system as follows:

$$
\dot{e}_{\ell}(t)=-\vec{s}_{\ell} e_{\ell}(t)+\sum_{z=1}^{n} \vec{\varphi}_{\ell z} \tilde{f}_{z}\left(e_{z}(t)\right)+\sum_{z=1}^{n} \vec{\phi}_{\ell z} \tilde{g}_{z}\left(e_{z}(t-\varepsilon(t))\right)+\Pi_{\ell}^{x}+u_{\ell} .
$$

After similar analysis to Case 3, one has

$$
\begin{aligned}
D^{+} V(t) & \leq\left(\vec{c}_{1}+\frac{\vec{c}_{2}}{2}\right)(W e(t))^{T} W e(t)+(W e(t))^{T} W(-\vec{S}-K) e(t) \\
& +\frac{\vec{c}_{2}}{2}(W e(t-\varepsilon(t)))^{T} W e(t-\varepsilon(t)) \\
& -\left(\sum_{\ell=1}^{n} w_{\ell}\left(\eta_{\ell}-\Pi_{\ell}\right)-\vec{M}_{1}-\vec{M}_{2}\right)\left|(W e(t))^{T}\right| \\
& \leq\left(-q+\vec{c}_{1}+\frac{\vec{c}_{2}}{2}\right)(W e(t))^{T} W e(t)+\frac{\vec{c}_{2}}{2}(W e(t-\varepsilon(t)))^{T} W e(t-\varepsilon(t)) \\
& \leq-\alpha V(t)+\beta V(t-\varepsilon(t)) .
\end{aligned}
$$

Finally, by (8)-(11) and according to Lemma 1, the following synchronization result can be derived:

$$
\|W e(t)\| \leq \sup _{-\mu \leq s \leq 0}\|W e(s)\| e^{-r t}
$$

where $r$ is the unique solution of $r=\alpha-\beta e^{r \mu}$. From the above, one can see that weighted sum complete synchronization is achieved with exponential synchronization rate $r$, and this completes the proof of Theorem 1.

Remark 2: In some published researches [46, 40, [54, in addition to meet Assumption $\left(H_{1}\right)$, the activation functions need to satisfy $f_{\ell}\left( \pm T_{\ell}\right)=g_{\ell}\left( \pm T_{\ell}\right)=$ 0 or $f_{\ell}(0)=g_{\ell}(0)=0$, but many functions do not have these properties. In this paper, we eliminate the strong conditions and only need to meet Assumption $\left(H_{1}\right)$, which is more reasonable and general.

Remark 3: Current synchronization researches on neural networks only focus on single node behavior. Actually in neural networks, there exists important combination behavior: weighted sum of node states. It is clear that weighted sum of node states is an important and essential step in function achievement of artificial neural networks [45]. In many researches on biological neural networks 43, 55, 56, it is indicated that weighted sum of node states plays a crucial role in information expression and coding; in contrast, single node can only express limited and rough information. Therefore, for function of neural networks, it is theoretically and practically important to study and control weighted sum of 185 node states.

Remark 4: In this section, only one controller is designed to achieve weighted sum complete synchronization. Obviously, designing one controller can reduce economic costs and is also easy to be implemented. Whereas, only one controller may make the entire control system fragile and paralyzed easily when 
the controller is subject to external deliberate attacks. Therefore, a new control scheme is needed to improve the situation.

\subsection{Weighted sum quasi-synchronization}

In the above section, weighted sum complete synchronization has been studied. As discussed before, the study of quasi-synchronization is also very meaningful for practical applications owing to the existence of interference factors in real environment. Therefore in this section, weighted sum quasi-synchronization will be investigated via designing a flexible aperiodically intermittent controller.

Note that in Theorem 1, only one controller is designed for the complete synchronization. Actually, for the proposed model, a more flexible control scheme can be devised because there exist multiple nodes but only one synchronization target. Considering the advantages of aperiodically intermittent control in saving control costs and based on this method, we will design a flexible control scheme to make the systems (1) and (4) achieve weighted sum quasisynchronization. Without loss of generality, the weights of the first $l$ nodes of the systems are assumed to be non-zero, and then the controller is designed as

$$
u_{m}(t)=\left\{\begin{array}{l}
r(t) \sum_{\ell=1}^{n} \frac{p_{m \ell}(t) w_{\ell}}{w_{m}} \sum_{z=1}^{n} k_{\ell z}^{*}(t)\left(x_{z}(t)-y_{z}(t)\right), m=1, \ldots, l \\
0, m=l+1, \ldots, n
\end{array}\right.
$$

where $k_{\ell z}^{*}(t)$ is control gain, $r(t)$ is intermittent control parameter and defined as follows: when $t \in T_{\text {work }}, r(t)=1$; when $t \in T_{\text {rest }}, r(t)=0$. Here, $T_{\text {work }}$ represents the work time interval $\left[t_{r}, \tau_{r}\right)$ and $T_{\text {rest }}$ represents the rest time interval $\left[\tau_{r}, t_{r+1}\right) . p_{m i}(t)$ is switching control parameter and taken as 0 or 1 ; it is worth noting that when $p_{m \ell}(t)$ meets $\sum_{m=1}^{l} \sum_{\ell=1}^{n} p_{m \ell}(t)=n$, after a simple calculation, we have

$$
W U=\left\{\begin{array}{l}
-W K^{*} e(t), t_{r} \leq t<\tau_{r} \\
0, \tau_{r} \leq t<t_{r+1}
\end{array}\right.
$$

where $K^{*}=\left(k_{\ell z}^{*}(t)\right)_{n \times n}$.

Remark 5: In current synchronization researches, controller is almost designed fixedly, that is, controller is invariable and unadjustable in synchronization process. For our synchronization model, the controller (13) is flexibly designed by introducing switching parameter $p_{m \ell}(t),(m=1, \ldots, l, \ell=1, \ldots, n)$. According to (13), it can be seen that we, by changing the value of $p_{m \ell}(t)$, can adjust the number of controllers or their positions in the networks. For example, 205 let $\sum_{\ell=1}^{n} p_{m \ell}(t)=n, m=j$ and $\sum_{\ell=1}^{n} p_{m \ell}(t)=0, m \neq j$, where $1 \leq j \leq l$ is an arbitrary integer, and then we obtain one controller but the controller position can be arbitrarily chosen. Also, multiple controllers can be obtained by correspondingly changing $p_{m \ell}(t)$. More importantly, $p_{m \ell}(t)$ is time-varying, which means that its value can be adjusted in real time to adapt to the new changes 210 or solve some new sudden control problems. Particularly, when the network is deliberately disturbed or attacked from the outside, timely adjusting controller 
by $p_{m \ell}(t)$ can help control system to deal with these problems, which is very important for the stability and security of the networks.

Theorem 2: By applying Assumptions $\left(H_{1}\right)$ and $\left(H_{2}\right)$, weighted sum quasisynchronization of drive system (1) and response system (4) is achieved under the controller (13), if parameter $p_{m \ell}(t)$ meet $\sum_{m=1}^{l} \sum_{\ell=1}^{n} p_{m \ell}(t)=n$, and the following conditions hold.

C1): $W\left(-S-K^{*}\right)=-q^{*} W$, where $q^{*}$ satisfies $\alpha^{*}=\min \left(q^{*}-\vec{c}_{1}-\frac{\vec{c}_{2}+1}{2}, q^{*}-\right.$ $\left.\overleftarrow{c}_{1}-\frac{\overleftarrow{c}_{2}+1}{2}\right)>\beta=\max \left(\frac{\overleftarrow{c}_{2}}{2}, \frac{\vec{c}_{2}}{2}\right)$

C2): $\alpha^{* *}=\max \left(\frac{1+\overleftarrow{c}_{2}}{2}-\frac{\lambda_{\min }(\overleftarrow{\Theta})}{\lambda_{\max }\left(W^{T} W\right)}, \frac{1+\vec{c}_{2}}{2}-\frac{\lambda_{\min }(\vec{\Theta})}{\lambda_{\max }\left(W^{T} W\right)}\right), \alpha^{* *}+\beta>0$.

where $\overleftarrow{\Theta}=\frac{1}{2}\left(\overleftarrow{S}^{T} W^{T} W+W^{T} W \overleftarrow{S}-W^{T} W \overleftarrow{\varphi}_{\varphi} \overleftarrow{\varphi}^{T} W^{T} W-A^{T} A\right), \vec{\Theta}=\frac{1}{2}\left(\vec{S}^{T} W^{T} W+\right.$ $\left.W^{T} W \vec{S}-W^{T} W \vec{\varphi} \vec{\varphi}^{T} W^{T} W-A^{T} A\right)$.

Proof: Introduce the following Lyapunov-Krasovskii function

$$
V(t)=\frac{1}{2}(W e(t))^{T} W e(t) .
$$

1) When $t_{r}<t \leq \tau_{r}$, the proof can be also divided into four cases.

Case 1: If $\left|x_{\ell}(t)\right| \leq T_{\ell},\left|y_{\ell}(t)\right| \leq T_{\ell}$ at time $t$, from (14), one has

$$
\begin{aligned}
D^{+} V(t) & =(W e(t))^{T} W \dot{e}(t) \\
& =(W e(t))^{T} W\left[\left(-\overleftarrow{S}-K^{*}\right) e(t)+\overleftarrow{\varphi} \tilde{f}(e(t))+\overleftarrow{\phi} \tilde{g}(e(t-\varepsilon(t)))\right]
\end{aligned}
$$

Note that the results similar to (6) and (7) of Theorem 1 are still valid in this proof; and based on these results, we have

$$
\begin{aligned}
D^{+} V(t) & \leq(W e(t))^{T} W\left(-\overleftarrow{S}-K^{*}\right) e(t)+\left(\overleftarrow{c}_{1}+\frac{\overleftarrow{c}_{2}}{2}\right)(W e(t))^{T} W e(t) \\
& +\frac{\overleftarrow{c}_{2}}{2}(W e(t-\varepsilon(t)))^{T} W e(t-\varepsilon(t))+\left(\overleftarrow{M}_{1}+\overleftarrow{M}_{2}\right)\left|(W e(t))^{T}\right| \\
& \leq-\left(q^{*}-\overleftarrow{c}_{1}-\frac{\overleftarrow{c}_{2}}{2}\right)(W e(t))^{T} W e(t) \\
& +\frac{\overleftarrow{c}_{2}}{2}(W e(t-\varepsilon(t)))^{T} W e(t-\varepsilon(t)) \\
& +\frac{1}{2}(W e(t))^{T} W e(t)+\frac{1}{2}\left(\overleftarrow{M}_{1}+\overleftarrow{M}_{2}\right)^{2}
\end{aligned}
$$

By the condition $C 1$ ), we have

$$
\begin{aligned}
D^{+} V(t) & \leq-\left(q^{*}-\overleftarrow{c}_{1}-\frac{\overleftarrow{c}_{2}+1}{2}\right)(W e(t))^{T} W e(t) \\
& +\frac{\overleftarrow{c}_{2}}{2}(W e(t-\varepsilon(t)))^{T} W e(t-\varepsilon(t))+\frac{1}{2}\left(\overleftarrow{M}_{1}+\overleftarrow{M}_{2}\right)^{2} \\
& \leq-\alpha^{*} V(t)+\beta V(t-\varepsilon(t))+\psi
\end{aligned}
$$


where $\psi=\max \left(\frac{1}{2}\left(\overleftarrow{M}_{1}+\overleftarrow{M}_{2}\right)^{2}, \frac{1}{2}\left(\vec{M}_{1}+\vec{M}_{2}+W \Pi\right)^{2}\right)$, and $\alpha^{*}$ and $\beta$ are defined 225 as mentioned earlier.

Case 2: If $\left|x_{\ell}(t)\right|>T_{\ell},\left|y_{\ell}(t)\right|>T_{\ell}$ at time $t$, after a similar inference to Case 1 , one obtains

$$
\begin{aligned}
D^{+} V(t) & \leq-\left(q^{*}-\vec{c}_{1}-\frac{\vec{c}_{2}+1}{2}\right)(W e(t))^{T} W e(t) \\
& +\frac{\vec{c}_{2}}{2}(W e(t-\varepsilon(t)))^{T} W e(t-\varepsilon(t))+\frac{1}{2}\left(\vec{M}_{1}+\vec{M}_{2}\right)^{2} \\
& \leq-\alpha^{*} V(t)+\beta V(t-\varepsilon(t))+\psi
\end{aligned}
$$

Case 3: If $\left|x_{\ell}(t)\right|>T_{\ell},\left|y_{\ell}(t)\right| \leq T_{\ell}$ at time $t$, the time derivative of $V(t)$ can be described as

$$
\begin{aligned}
D^{+} V(t) & =(W e(t))^{T} W\left[\left(-\vec{S}-K^{*}\right) e(t)+\vec{\varphi} \tilde{f}(e(t))\right. \\
& \left.+\vec{\phi} \tilde{g}(e(t-\varepsilon(t)))+\Pi^{y}\right]
\end{aligned}
$$

Noting that $(W e(t))^{T} W \Pi^{y} \leq W \Pi\left|(W e(t))^{T}\right|$ and according to the condition C1), then one has

$$
\begin{aligned}
D^{+} V(t) & \leq(W e(t))^{T} W\left(-\vec{S}-K^{*}\right) e(t)+\left(\vec{c}_{1}+\frac{\vec{c}_{2}}{2}\right)(W e(t))^{T} W e(t) \\
& +\frac{\vec{c}_{2}}{2}(W e(t-\varepsilon(t)))^{T} W e(t-\varepsilon(t)) \\
& +\left(\vec{M}_{1}+\vec{M}_{2}+W \Pi\right)\left|(W e(t))^{T}\right| \\
& \leq-\left(q^{*}-\vec{c}_{1}-\frac{\vec{c}_{2}}{2}\right)(W e(t))^{T} W e(t) \\
& +\frac{\overrightarrow{c_{2}}}{2}(W e(t-\varepsilon(t)))^{T} W e(t-\varepsilon(t)) \\
& +\frac{1}{2}(W e(t))^{T} W e(t)+\frac{1}{2}\left(\vec{M}_{1}+\vec{M}_{2}+W \Pi\right)^{2} \\
& \leq-\alpha^{*} V(t)+\beta V(t-\varepsilon(t))+\psi
\end{aligned}
$$

Case 4: If $\left|x_{\ell}(t)\right| \leq T_{\ell},\left|y_{\ell}(t)\right|>T_{\ell}$ at time $t$, similar to Case 3 , it is obtained that

$$
\begin{aligned}
D^{+} V(t) & =(W e(t))^{T} W\left[\left(-\vec{S}-K^{*}\right) e(t)+\vec{\varphi} \tilde{f}(e(t))\right. \\
& \left.+\vec{\phi} \tilde{g}(e(t-\varepsilon(t)))+\Pi^{x}\right] \\
& \leq-\left(q^{*}-\vec{c}_{1}-\frac{\vec{c}_{2}+1}{2}\right)(W e(t))^{T} W e(t) \\
& +\frac{\vec{c}_{2}}{2}(W e(t-\varepsilon(t)))^{T} W e(t-\varepsilon(t)) \\
& +\frac{1}{2}\left(\vec{M}_{1}+\vec{M}_{2}+W \Pi\right)^{2} \\
& \leq-\alpha^{*} V(t)+\beta V(t-\varepsilon(t))+\psi
\end{aligned}
$$


Thus, according to (15)-(18), condition C1) and Lemma 2, we obtain

$$
V(t) \leq V_{\mu}\left(t_{r}\right) e^{-\varpi\left(t-t_{r}\right)}+\varsigma, t_{r}<t \leq \tau_{r}
$$

where $\varpi$ is the unique positive solution of $-\alpha^{*}+\beta e^{\varpi \mu}+\varpi=0$, and $\varsigma=\psi / \varpi$.

2) When $\tau_{r}<t \leq t_{r+1}$, in Case 1 , one has

$$
\begin{aligned}
D^{+} V(t) & =(W e(t))^{T} W[-\overleftarrow{S} e(t)+\overleftarrow{\varphi} \tilde{f}(e(t))+\overleftarrow{\phi} \tilde{g}(e(t-\varepsilon(t)))] \\
& \leq-\frac{1}{2} e(t)^{T}\left(\overleftarrow{S}^{T} W^{T} W+W^{T} W \overleftarrow{S}\right) e(t) \\
& +(W e(t))^{T} W[\overleftarrow{\varphi} \tilde{f}(e(t))+\overleftarrow{\phi} \tilde{g}(e(t-\varepsilon(t)))]
\end{aligned}
$$

From Lemma 5 and Assumption $\left(H_{1}\right)$, we obtain

$$
\begin{aligned}
& (W e(t))^{T} W \overleftarrow{\varphi} \tilde{f}(e(t)) \leq \frac{1}{2} e(t)^{T} W^{T} W \overleftarrow{\varphi} \overleftarrow{\varphi}^{T} W^{T} W e(t)+\frac{1}{2} \tilde{f}(e(t))^{T} \tilde{f}(e(t)) \\
& \leq \frac{1}{2} e(t)^{T} W^{T} W \overleftarrow{\varphi}^{T}{ }^{T} W^{T} W e(t)+\frac{1}{2} e(t)^{T} A^{T} A e(t)
\end{aligned}
$$

Then, by (7), we have

$$
\begin{aligned}
D^{+} V(t) & \leq e(t)^{T}\left[-\frac{1}{2}\left(\overleftarrow{S}^{T} W^{T} W+W^{T} W \overleftarrow{S}\right)+\frac{1}{2} W^{T} W \overleftarrow{\varphi}^{T} \overleftarrow{\varphi}^{T} W^{T} W\right. \\
& \left.+\frac{1}{2} A^{T} A\right] e(t)+\frac{1+\overleftarrow{c}_{2}}{2}(W e(t))^{T} W e(t) \\
& +\frac{\overleftarrow{c}_{2}}{2}(W e(t-\varepsilon(t)))^{T} W e(t-\varepsilon(t))+\frac{1}{2} \overleftarrow{M}_{2}{ }^{2}
\end{aligned}
$$

Note that

$$
\begin{aligned}
e(t)^{T}[ & \left.-\frac{1}{2}\left(\overleftarrow{S}^{T} W^{T} W+W^{T} W \overleftarrow{S}\right)+\frac{1}{2} W^{T} W \overleftarrow{\varphi}^{\overleftarrow{\varphi}^{T}} W^{T} W+\frac{1}{2} A^{T} A\right] e(t) \\
& \left.\leq-\frac{\lambda_{\min }(\overleftarrow{\Theta})}{\lambda_{\max }\left(W^{T} W\right)}\right) e(t)^{T} W^{T} W e(t)
\end{aligned}
$$

where $\overleftarrow{\Theta}$ has been defined in Theorem 2

Thus

$$
\begin{aligned}
D^{+} V(t) & \leq\left(\frac{1+\overleftarrow{c}_{2}}{2}-\frac{\lambda_{\min }(\overleftarrow{\Theta})}{\lambda_{\max }\left(W^{T} W\right)}\right) e(t)^{T} W^{T} W e(t) \\
& +\frac{\overleftarrow{c_{2}}}{2}(W e(t-\varepsilon(t)))^{T} W e(t-\varepsilon(t))+\frac{1}{2} \overleftarrow{M}_{2}{ }^{2} \\
& \leq-\alpha^{* *} V(t)+\beta V(t-\varepsilon(t))+\psi^{*}
\end{aligned}
$$

where $\psi^{*}=\max \left(\frac{1}{2} \overleftarrow{M}_{2}^{2}, \frac{1}{2} \vec{M}_{2}^{2}, \frac{1}{2}\left(\vec{M}_{2}+W \Pi\right)^{2}\right)$, and $\alpha^{* *}, \beta$ have been defined in Theorem 2 . 
In Case 2, similarly, it is inferred that

$$
\begin{aligned}
D^{+} V(t) & \leq\left(\frac{1+\vec{c}_{2}}{2}-\frac{\lambda_{\min }(\vec{\Theta})}{\lambda_{\max }\left(W^{T} W\right)}\right) e(t)^{T} W^{T} W e(t) \\
& +\frac{\vec{c}_{2}}{2}(W e(t-\varepsilon(t)))^{T} W e(t-\varepsilon(t))+\frac{1}{2} \vec{M}_{2}^{2} \\
& \leq-\alpha^{* *} V(t)+\beta V(t-\varepsilon(t))+\psi^{*}
\end{aligned}
$$

In Case 3, we have

$$
\begin{aligned}
D^{+} V(t) & =(W e(t))^{T} W\left[-\vec{S} e(t)+\vec{\varphi} \tilde{f}(e(t))+\vec{\phi} \tilde{g}(e(t-\varepsilon(t)))+\Pi^{y}\right] \\
& \leq e(t)^{T}\left[-\frac{1}{2}\left(\vec{S}^{T} W^{T} W+W^{T} W \vec{S}\right)+\frac{1}{2} W^{T} W \vec{\varphi} \vec{\varphi}^{T} W^{T} W\right. \\
& \left.+\frac{1}{2} A^{T} A\right] e(t)+\frac{\vec{c}_{2}}{2}(W e(t-\varepsilon(t)))^{T} W e(t-\varepsilon(t)) \\
& +\frac{\vec{c}_{2}}{2}(W e(t))^{T} W e(t)+\left(\vec{M}_{2}+\Pi\right)\left|(W e(t))^{T}\right| \\
& \leq\left(\frac{1+\vec{c}_{2}}{2}-\frac{\lambda_{\min }(\vec{\Theta})}{\lambda_{\max }\left(W^{T} W\right)}\right) e(t)^{T} W^{T} W e(t) \\
& +\frac{\vec{c}_{2}}{2}(W e(t-\varepsilon(t)))^{T} W e(t-\varepsilon(t))+\frac{1}{2}\left(\vec{M}_{2}+W \Pi\right)^{2} \\
& \leq-\alpha^{* *} V(t)+\beta V(t-\varepsilon(t))+\psi^{*}
\end{aligned}
$$

In Case 4, similarly, we obtain

$$
\begin{aligned}
D^{+} V(t) & =(W e(t))^{T} W\left[-\vec{S} e(t)+\vec{\varphi} \tilde{f}(e(t))+\vec{\phi} \tilde{g}(e(t-\varepsilon(t)))+\Pi^{x}\right] \\
& \leq\left(\frac{1+\vec{c}_{2}}{2}-\frac{\lambda_{\min }(\vec{\Theta})}{\lambda_{\max }\left(W^{T} W\right)}\right) e(t)^{T} W^{T} W e(t) \\
& +\frac{\vec{c}_{2}}{2}(W e(t-\varepsilon(t)))^{T} W e(t-\varepsilon(t))+\frac{1}{2}\left(\vec{M}_{2}+W \Pi\right)^{2} \\
& \leq-\alpha^{* *} V(t)+\beta V(t-\varepsilon(t))+\psi^{*}
\end{aligned}
$$

Let $\omega=\alpha^{* *}+\beta$, and according to (19)-(22), condition C2) and Lemma 3, we obtain

$$
V(t) \leq\left(V_{\mu}\left(\tau_{r}\right)+\xi\right) e^{\omega\left(t-\tau_{r}\right)}-\xi, \tau_{r}<t \leq t_{r+1}
$$

230 where $\xi=\psi^{*} / \omega$.

Finally, based on Lemma (4), we have

$$
V(t) \leq V_{\mu}(0) e^{h} e^{-(h / \sigma) t}+\frac{\rho}{1-e^{-h}}+\varsigma, t>0 .
$$

where $h=h_{1}-h_{2}>0, h_{1}=\varpi(\theta-\mu), h_{2}=\omega(\sigma-\theta), \theta=\inf _{r}\left(\tau_{r}-t_{r}\right), \sigma=$ $\sup _{r}\left(t_{r+1}-t_{r}\right), \rho=(\varsigma+\xi) e^{h_{2}}-\xi$.

Thus, it can be inferred that

$$
|W e(t)| \leq \sqrt{\frac{2 \rho}{1-e^{-h}}+2 \varsigma}, \mathrm{t} \rightarrow+\infty
$$


which indicates that drive-response systems (1) and (4) obtain weighted sum quasi-synchronization with exponential synchronization rate and error range $\sqrt{2 \rho /\left(1-e^{-h}\right)+2 \varsigma}$. This completes the proof.

Remark 6: It is worth noting that the aperiodically intermittent control can achieve complete synchronization of general nonlinear dynamic system [41], [57, but cannot guarantee complete synchronization of memristive neural networks. The main reason is that unlike general nonlinear dynamic networks, memristive neural networks are state-dependent switching systems, that is, the values of the network coefficients depend on node state. This will result in mismatches of corresponding parameters of drive-response systems particularly when driveresponse systems have different initial values. Over time, parameter mismatches will occur and produce unwanted disturbances and errors in synchronization process. Because during the rest time interval $\left[\tau_{r}, t_{r+1}\right)$, these disturbances and errors cannot be guaranteed to be eliminated, intermittent control methods are currently used to ensure quasi-synchronization of memristive neural networks.

\subsection{Illustrative examples}

In this section, two numerical simulations will be given to check the validity of the above theoretical results.

Example 1: consider the following 2-D memristive coupled neural networks:

$$
\left\{\begin{aligned}
\dot{x}_{1}(t)= & -s_{1}\left(x_{1}(t)\right) x_{1}(t)+\varphi_{11}\left(x_{1}(t)\right) f\left(x_{1}(t)\right) \\
& +\varphi_{12}\left(x_{1}(t)\right) f\left(x_{2}(t)\right)+\phi_{11}\left(x_{1}(t)\right) g\left(x_{1}(t-\varepsilon(t))\right) \\
& +\phi_{12}\left(x_{1}(t)\right) g\left(x_{2}(t-\varepsilon(t))\right)+I_{1} \\
\dot{x}_{2}(t)= & -s_{2}\left(x_{2}(t)\right) x_{2}(t)+\varphi_{21}\left(x_{2}(t)\right) f\left(x_{1}(t)\right) \\
& +\varphi_{22}\left(x_{2}(t)\right) f\left(x_{2}(t)\right)+\phi_{21}\left(x_{2}(t)\right) g\left(x_{1}(t-\varepsilon(t))\right) \\
& +\phi_{22}\left(x_{2}(t)\right) g\left(x_{2}(t-\varepsilon(t))\right)+I_{2}
\end{aligned}\right.
$$

where

$$
\begin{gathered}
s_{1}\left(x_{1}(t)\right)=\left\{\begin{array}{l}
1,\left|x_{1}(t)\right| \leq 1.1 \\
1,\left|x_{1}(t)\right|>1.1
\end{array} \quad s_{2}\left(x_{2}(t)\right)=\left\{\begin{array}{l}
1,\left|x_{2}(t)\right| \leq 1.1 \\
1,\left|x_{2}(t)\right|>1.1
\end{array}\right.\right. \\
\varphi_{11}\left(x_{1}(t)\right)=\left\{\begin{array}{l}
-0.4,\left|x_{1}(t)\right| \leq 1.1 \\
-0.5,\left|x_{1}(t)\right|>1.1
\end{array} \quad \varphi_{12}\left(x_{1}(t)\right)=\left\{\begin{array}{l}
1.2,\left|x_{1}(t)\right| \leq 1.1 \\
1.3,\left|x_{1}(t)\right|>1.1
\end{array}\right.\right. \\
\varphi_{21}\left(x_{2}(t)\right)=\left\{\begin{array}{l}
1.6,\left|x_{2}(t)\right| \leq 1.1 \\
1.7,\left|x_{2}(t)\right|>1.1
\end{array} \quad \varphi_{22}\left(x_{2}(t)\right)=\left\{\begin{array}{l}
-0.55,\left|x_{2}(t)\right| \leq 1.1 \\
-0.45,\left|x_{2}(t)\right|>1.1
\end{array}\right.\right. \\
\phi_{11}\left(x_{1}(t)\right)=\left\{\begin{array}{l}
-0.3,\left|x_{1}(t)\right| \leq 1.1 \\
-0.5,\left|x_{1}(t)\right|>1.1
\end{array} \quad \phi_{12}\left(x_{1}(t)\right)=\left\{\begin{array}{l}
2.5,\left|x_{1}(t)\right| \leq 1.1 \\
2.1,\left|x_{1}(t)\right|>1.1
\end{array}\right.\right. \\
\phi_{21}\left(x_{2}(t)\right)=\left\{\begin{array}{l}
2.4,\left|x_{2}(t)\right| \leq 1.1 \\
2.3,\left|x_{2}(t)\right|>1.1
\end{array} \quad \phi_{22}\left(x_{2}(t)\right)=\left\{\begin{array}{l}
-0.2,\left|x_{2}(t)\right| \leq 1.1 \\
-0.3,\left|x_{2}(t)\right|>1.1 .
\end{array}\right.\right.
\end{gathered}
$$


Correspondingly, response system (4) with controller is written as

$$
\left\{\begin{aligned}
\dot{y}_{1}(t) & =-s_{1}\left(y_{1}(t)\right) y_{1}(t)+\varphi_{11}\left(y_{1}(t)\right) f\left(y_{1}(t)\right) \\
& +\varphi_{12}\left(y_{1}(t)\right) f\left(y_{2}(t)\right)+\phi_{11}\left(y_{1}(t)\right) g\left(y_{1}(t-\varepsilon(t))\right) \\
& +\phi_{12}\left(y_{1}(t)\right) g\left(y_{2}(t-\varepsilon(t))\right) \\
& +k_{11}(t)\left(x_{1}(t)-y_{1}(t)\right)+k_{12}(t)\left(x_{2}(t)-y_{2}(t)\right) \\
& +\eta_{1} \operatorname{sign}\left(w_{1}\left(x_{1}(t)-y_{1}(t)\right)+w_{2}\left(x_{2}(t)-y_{2}(t)\right)\right) \\
& +\frac{w_{2}}{w_{1}}\left[k_{21}(t)\left(x_{1}(t)-y_{1}(t)\right)+k_{22}(t)\left(x_{2}(t)-y_{2}(t)\right)\right. \\
& \left.+\eta_{2} \operatorname{sign}\left(w_{1}\left(x_{1}(t)-y_{1}(t)\right)+w_{2}\left(x_{2}(t)-y_{2}(t)\right)\right)\right]+I_{1} \\
\dot{y}_{2}(t) & =-s_{2}\left(y_{2}(t)\right) y_{2}(t)+\varphi_{21}\left(y_{2}(t)\right) f\left(y_{1}(t)\right) \\
& +\varphi_{22}\left(y_{2}(t)\right) f\left(y_{2}(t)\right)+\phi_{21}\left(y_{2}(t)\right) g\left(y_{1}(t-\varepsilon(t))\right) \\
& +\phi_{22}\left(y_{2}(t)\right) g\left(y_{2}(t-\varepsilon(t))\right)+I_{2} .
\end{aligned}\right.
$$

Let the nonlinear activation functions $f_{i}(x)=g_{i}(x)=\sin (x), i=1,2$, time delay $\varepsilon(t)=(e(t)-1) /(e(t)+1)$, and external inputs $I_{1}=I_{2}=0$. It is easy to get that $l_{\ell}=a_{\ell}=1, \ell=1,2$. By choosing $W=\left(\begin{array}{l}1 \\ 2\end{array}\right), \vec{c}_{1}=\overleftarrow{c}_{1}=0.1, \vec{c}_{2}=\overleftarrow{c}_{2}=$ $0.2, K=\left[\begin{array}{ll}0 & 2 \\ 1 & 1\end{array}\right], \eta_{1}=\eta_{2}=7$, after a simple calculation, we obtain $q=3$, $\alpha=2.8>\beta=0.1$ and $w_{1} \eta_{1}+w_{2} \eta_{2}=21>\gamma=20.7$, which verifies Theorem 1. In the following, MATLAB simulation will manifest the correctness of the derived results.

Take the initial values of the systems $x(t)=(-3,7)^{T}$ and $y(t)=(0,-1.3)^{T}$, where $t \in[-1,0]$. Fig. 3 presents the phase trajectories of memristive neural network model and Fig. 4 shows the change of a coefficient $\varphi_{12}\left(x_{1}(t)\right)$. The node trajectories of the drive and response systems are illustrated by Figs. 5 and 6 , from which one can see that nodes $x_{1}(t)$ and $x_{2}(t)$ are not synchronized with the corresponding nodes $y_{1}(t)$ and $y_{2}(t)$. Fig. 7 shows that the node errors $e_{1}(t)$ and $e_{2}(t)$ do not approach to zero as time evolves, but the weighted error sum, as shown in Fig. 8, clearly tends to zero, which confirms the proposed results in Theorem 1.

Example 2: consider the memristive neural networks:

$$
\left\{\begin{aligned}
c \dot{x}_{1}(t) & =-s_{1}\left(x_{1}(t)\right) x_{1}(t)+\varphi_{11}\left(x_{1}(t)\right) f\left(x_{1}(t)\right) \\
& +\varphi_{12}\left(x_{1}(t)\right) f\left(x_{2}(t)\right)+\phi_{11}\left(x_{1}(t)\right) g\left(x_{1}(t-\varepsilon(t))\right) \\
& +\phi_{12}\left(x_{1}(t)\right) g\left(x_{2}(t-\varepsilon(t))\right)+I_{1} \\
\dot{x}_{2}(t) & =-s_{2}\left(x_{2}(t)\right) x_{2}(t)+\varphi_{21}\left(x_{2}(t)\right) f\left(x_{1}(t)\right) \\
& +\varphi_{22}\left(x_{2}(t)\right) f\left(x_{2}(t)\right)+\phi_{21}\left(x_{2}(t)\right) g\left(x_{1}(t-\varepsilon(t))\right) \\
& +\phi_{22}\left(x_{2}(t)\right) g\left(x_{2}(t-\varepsilon(t))\right)+I_{2}
\end{aligned}\right.
$$

where

$$
s_{1}\left(x_{1}(t)\right)=\left\{\begin{array}{l}
1,\left|x_{1}(t)\right| \leq 1.1 \\
1,\left|x_{1}(t)\right|>1.1
\end{array} \quad s_{2}\left(x_{2}(t)\right)=\left\{\begin{array}{l}
1,\left|x_{2}(t)\right| \leq 1.1 \\
1,\left|x_{2}(t)\right|>1.1
\end{array}\right.\right.
$$




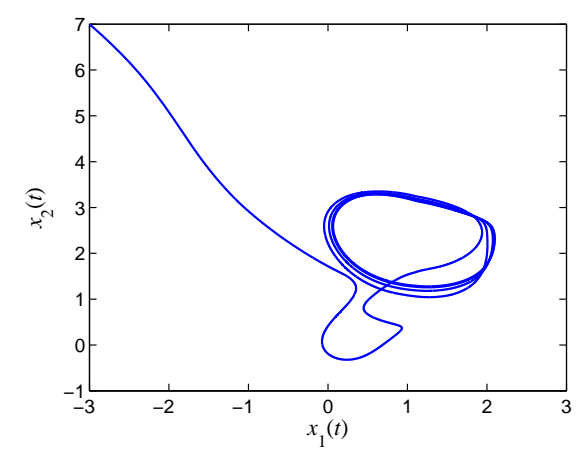

Figure 3: Phase portrait of memristive neural network model.

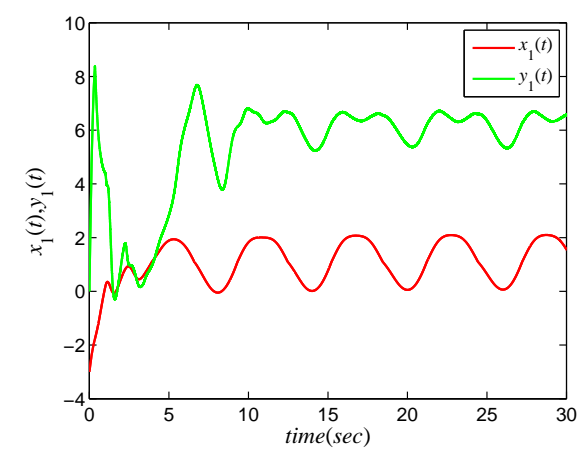

Figure 5: States of $x_{1}(t)$ and $y_{1}(t)$.

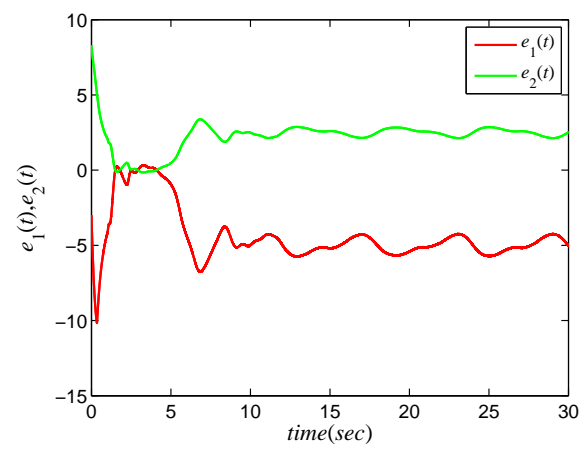

Figure 7: Errors $e_{1}(t)$ and $e_{2}(t)$ under the controller.

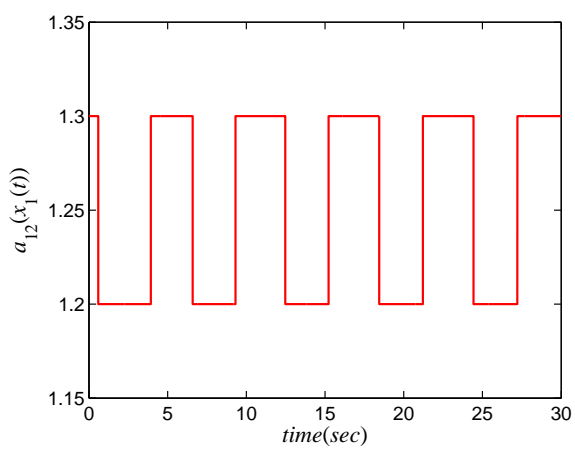

Figure 4: Memristive connection coefficient $\varphi_{12}\left(x_{1}(t)\right)$.

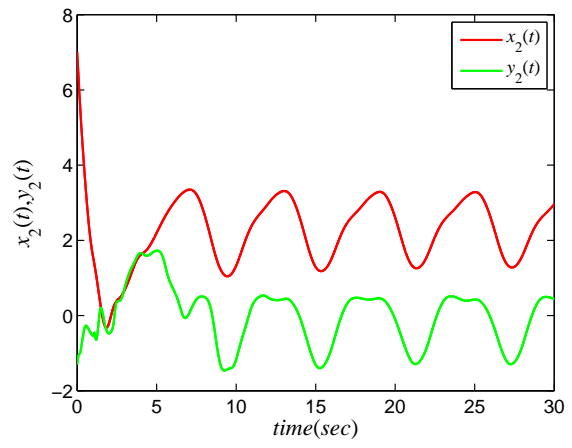

Figure 6: States of $x_{2}(t)$ and $y_{2}(t)$.

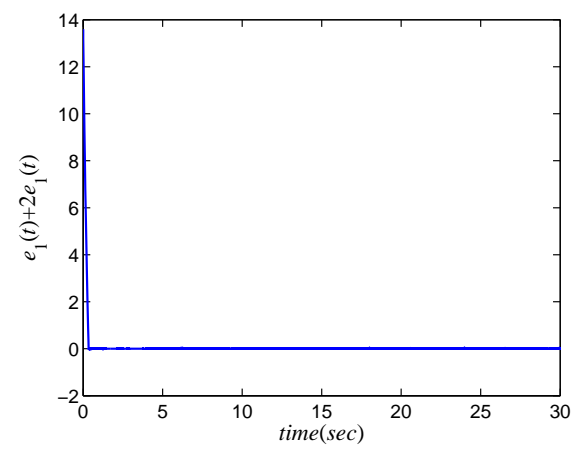

Figure 8: Weighted error sum $e_{1}(t)+2 e_{2}(t)$. 


$$
\begin{aligned}
& \varphi_{11}\left(x_{1}(t)\right)=\left\{\begin{array}{l}
-5,\left|x_{1}(t)\right| \leq 1.1 \\
-5.1,\left|x_{1}(t)\right|>1.1
\end{array} \quad \varphi_{12}\left(x_{1}(t)\right)=\left\{\begin{array}{l}
3.1,\left|x_{1}(t)\right| \leq 1.1 \\
3.2,\left|x_{1}(t)\right|>1.1
\end{array}\right.\right. \\
& \varphi_{21}\left(x_{2}(t)\right)=\left\{\begin{array}{l}
2.55,\left|x_{2}(t)\right| \leq 1.1 \\
2.6,\left|x_{2}(t)\right|>1.1
\end{array} \quad \varphi_{22}\left(x_{2}(t)\right)=\left\{\begin{array}{l}
-1.5,\left|x_{2}(t)\right| \leq 1.1 \\
-1.6,\left|x_{2}(t)\right|>1.1
\end{array}\right.\right. \\
& \phi_{11}\left(x_{1}(t)\right)=\left\{\begin{array}{l}
-6.1,\left|x_{1}(t)\right| \leq 1.1 \\
-6.2,\left|x_{1}(t)\right|>1.1
\end{array} \quad \phi_{12}\left(x_{1}(t)\right)=\left\{\begin{array}{l}
5.15,\left|x_{1}(t)\right| \leq 1.1 \\
5.2,\left|x_{1}(t)\right|>1.1
\end{array}\right.\right. \\
& \phi_{21}\left(x_{2}(t)\right)=\left\{\begin{array}{l}
3.0,\left|x_{2}(t)\right| \leq 1.1 \\
3.1,\left|x_{2}(t)\right|>1.1
\end{array} \quad \phi_{22}\left(x_{2}(t)\right)=\left\{\begin{array}{l}
-2.5,\left|x_{2}(t)\right| \leq 1.1 \\
-2.6,\left|x_{2}(t)\right|>1.1 .
\end{array}\right.\right.
\end{aligned}
$$

In the following, the effectiveness of another proposed control scheme will be proved. It is worth noting that to save control cost, switching control matrix $P$ remains invariant until something motivational happens such as a big synchronization error. Thus, at first, an experiment is given where $P$ is invariant. Then, to verify anti-interference ability of the control scheme, another simulation experiment is given where an attack happens and $P$ is adjusted to ensure synchronization achievement.

At first, we choose and keep

$$
P=\left[\begin{array}{ll}
1 & 1 \\
0 & 0
\end{array}\right]
$$

Then, the response system (4) can be written as

$$
\left\{\begin{aligned}
\dot{y}_{1}(t) & =-s_{1}\left(y_{1}(t)\right) y_{1}(t)+\varphi_{11}\left(y_{1}(t)\right) f\left(y_{1}(t)\right) \\
& +\varphi_{12}\left(y_{1}(t)\right) f\left(y_{2}(t)\right)+\phi_{11}\left(y_{1}(t)\right) g\left(y_{1}(t-\varepsilon(t))\right) \\
& +\phi_{12}\left(y_{1}(t)\right) g\left(y_{2}(t-\varepsilon(t))\right) \\
& +r(t) k_{11}(t)\left(x_{1}(t)-y_{1}(t)\right)+r(t) k_{12}(t)\left(x_{2}(t)-y_{2}(t)\right) \\
& +\frac{w_{2}}{w_{1}} r(t)\left[k_{21}(t)\left(x_{1}(t)-y_{1}(t)\right)+k_{22}(t)\left(x_{2}(t)-y_{2}(t)\right)\right]+I_{1} \\
\dot{y}_{2}(t) & =-s_{2}\left(y_{2}(t)\right) y_{2}(t)+\varphi_{21}\left(y_{2}(t)\right) f\left(y_{1}(t)\right) \\
& +\varphi_{22}\left(y_{2}(t)\right) f\left(y_{2}(t)\right)+\phi_{21}\left(y_{2}(t)\right) g\left(y_{1}(t-\varepsilon(t))\right) \\
& +\phi_{22}\left(y_{2}(t)\right) g\left(y_{2}(t-\varepsilon(t))\right)+I_{2} .
\end{aligned}\right.
$$

Consider time delay $\varepsilon(t)=e(t) /(1+e(t))<1$ and let the nonlinear activation functions $f(x)=g(x)=\left(\sin x_{1}, \sin x_{2}\right)$. We can derive $l_{1}=l_{2}=a_{\ell}=1, \ell=$ $1, \ldots, n$. By choosing $\vec{c}_{\ell}=\overleftarrow{c}_{\ell}=0.1, \ell=1,2, K=\left[\begin{array}{ll}2 & 4 \\ 2 & 4\end{array}\right]$,it can be calculated that $\alpha^{*}=6.35>\beta=0.05, \alpha^{* *}+\beta=0.7>0, \psi=0.85, \psi^{*}=0.61, q^{*}=$ $7, \varpi=3.9, \omega=0.7, \varsigma=0.28, \xi=0.86$, and these results guarantee the conditions in Theorem 2. For convenience, choose fixed intermittent control interval $\tau_{\ell}-t_{\ell}=3.9, t_{\ell+1}-t_{\ell}=4, \ell=1,2,3, \ldots$ Then, we can compute $h_{1}=11.31, h_{2}=0.07, h=11.24, \rho=0.30, \sqrt{2 \rho /\left(1-e^{-h}\right)+2 \varsigma}=1.01$.

With the initial values $x(t)=(-1,2)^{T}$ and $y(t)=(0,-1.3)^{T}, t \in[-1,0]$, time trajectories of states of $x(t)$ and $y(t)$ can be presented in Figs. 9 and 10, and 

in Theorem 2, the systems can also achieve weighted sum quasi-synchronization.

Remark 8: Compared to the existing literatures which employ linear matrix inequalities (LMIs) to derive some synchronization conditions, such as 58, and [59, our conditions are directly obtained from the network parameters and can be verified more easily.

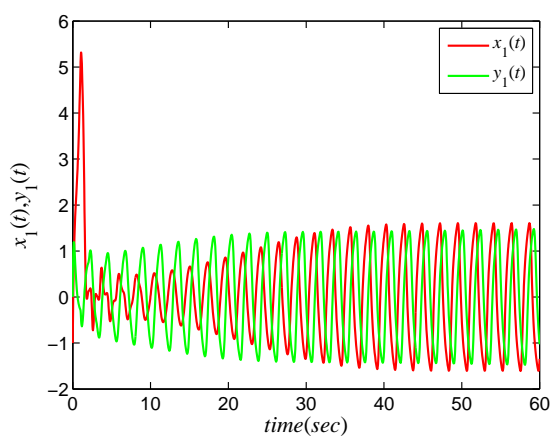

Figure 9: States of $x_{1}(t)$ and $y_{1}(t)$.

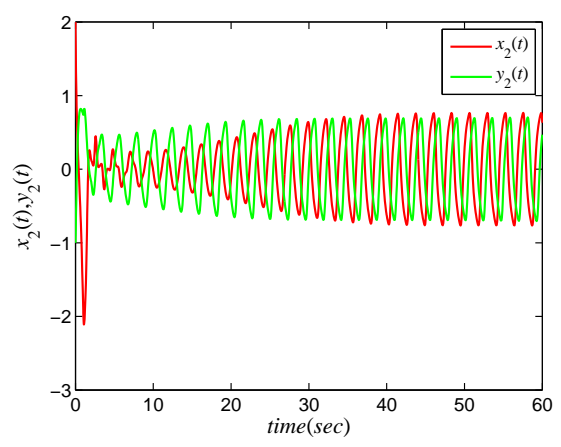

Figure 10: States of $x_{2}(t)$ and $y_{2}(t)$. 


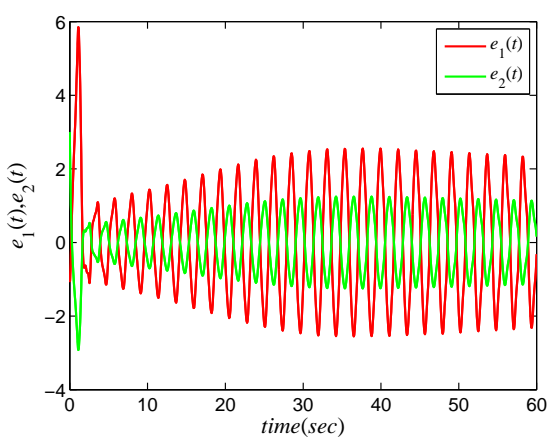

Figure 11: Errors $e_{1}(t)$ and $e_{2}(t)$ under the controller.

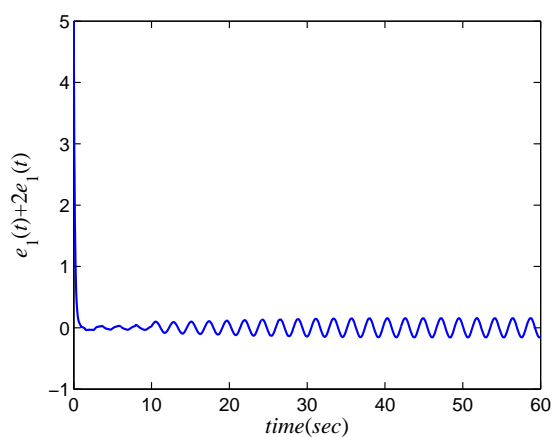

Figure 13: The weighted error sums under the attack.

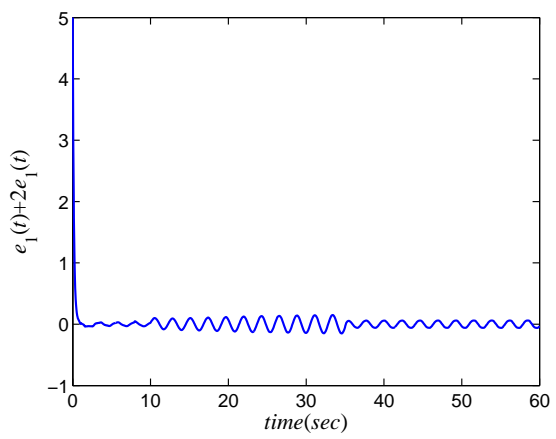

Figure 15: The weighted error sums after adding new controllers at the time $t=35 \mathrm{~s}$

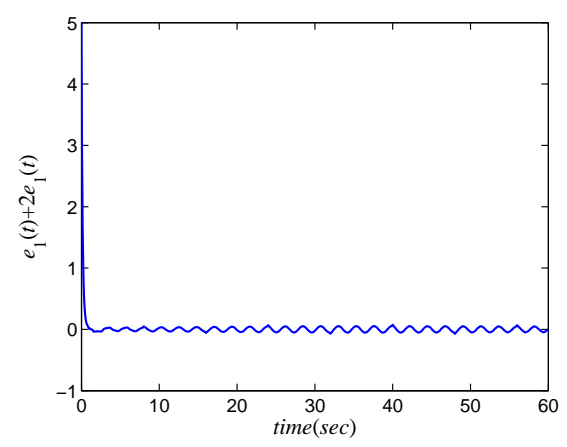

Figure 12: Weighted error sum $e_{1}(t)+2 e_{2}(t)$.

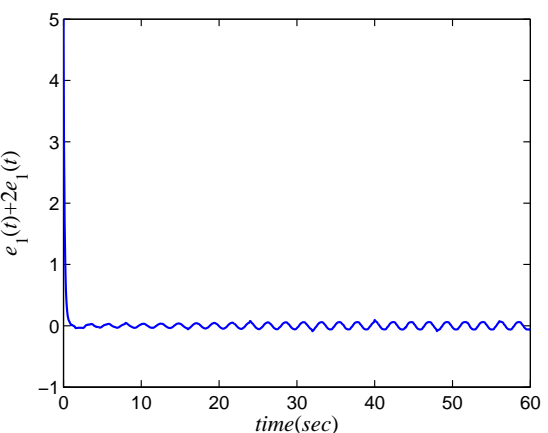

Figure 14: The weighted error sums after adding new controllers at the time $t=10 \mathrm{~s}$. 


\section{Conclusion}

In this paper, different to current node-to-node synchronization model, a new weighted sum synchronization of memristive neural networks has been studied. And aimed at the proposed synchronization model, two control methods are designed. First, in order to achieve weighted sum complete synchronization, a state feedback controller is designed and based on Lyapunov function and Halanary inequality, several sufficient conditions of weighted sum complete synchronization have been derived. Then, a flexible aperiodically intermittent control method is designed to investigat weighted sum quasi-synchronization.

325 Moreover, by introducing some switching parameters, this method can improve the anti-interference ability of control system, which is very important for the network stability. Meanwhile, the sufficient conditions of quasi-synchronization and the error level have also been presented. Finally, numerical simulations verify the theoretical analysis precisely. In this paper, the new synchroniza330 tion model is achieved but with exponential synchronization rate. In practical applications, there always exists a time-efficiency requirement for synchronization implementation. Thus it will be very meaningful to study finite-time and fixed-time synchronization problems in our future works.

\section{References}

[1] L. Chua, Memristor-the missing circuit element, IEEE Transactions on Circuit Theory 18 (5) (1971) 507-519.

[2] D. B. Strukov, G. S. Snider, D. R. Stewart, R. S. Williams, The missing memristor found, Nature 453 (7191) (2008) 80.

[3] K. D. Cantley, A. Subramaniam, H. J. Stiegler, R. A. Chapman, E. M. Vogel, Hebbian learning in spiking neural networks with nanocrystalline silicon tfts and memristive synapses, IEEE Transactions on Nanotechnology 10 (5) (2011) 1066-1073.

[4] Y. V. Pershin, M. Di Ventra, Experimental demonstration of associative memory with memristive neural networks, Neural Networks 23 (7) (2010) $881-886$.

[5] F. Yu, L. Li, B. He, L. Liu, S. Qian, Y. Huang, S. Cai, Y. Song, Q. Tang, Q. Wan, et al., Design and fpga implementation of a pseudorandom number generator based on a four-wing memristive hyperchaotic system and bernoulli map, IEEE Access (2019), doi: 10.1109/ACCESS.2019.2956573.

[6] C. Wang, X. Liu, H. Xia, Multi-piecewise quadratic nonlinearity memristor and its $2 \mathrm{n}$-scroll and $2 \mathrm{n}+1$-scroll chaotic attractors system, Chaos: An Interdisciplinary Journal of Nonlinear Science 27 (3) (2017) 033114. 
[7] F. Yu, L. Liu, B. He, Y. Huang, C. Shi, S. Cai, Y. Song, S. Du, Q. Wan, Analysis and fpga realization of a novel 5d hyperchaotic four-wing memristive system, active control synchronization, and secure communication application, Complexity (2019), doi: 10.1155/2019/4047957.

[8] L. Ding, W. X. Zheng, Network-based practical consensus of heterogeneous nonlinear multiagent systems, IEEE Transactions on Cybernetics 47 (8) (2016) 1841-1851.

[9] Y. Wang, T. Bian, J. Xiao, C. Wen, Global synchronization of complex dynamical networks through digital communication with limited data rate, IEEE Transactions on Neural Networks and Learning Systems 26 (10) (2015) 2487-2499.

[10] J. Jin, L. Zhao, M. Li, F. Yu, Z. Xi, Improved zeroing neural networks for finite time solving nonlinear equations, Neural Computing and Applications, (2019), doi:10.1007/s00521-019-04622-x.

[11] Q. Xiao, Z. Huang, Z. Zeng, Passivity analysis for memristor-based inertial neural networks with discrete and distributed delays, IEEE Transactions on Systems, Man, and Cybernetics: Systems 49 (2) (2017) 375-385.

[12] L. Wang, H. He, Z. Zeng, C. Hu, Global stabilization of fuzzy memristorbased reaction-diffusion neural networks, IEEE Transactions on Cybernetics (2019),doi:10.1109/TCYB.2019.2949468.

[13] W. Yao, C. Wang, J. Cao, Y. Sun, C. Zhou, Hybrid multisynchronization of coupled multistable memristive neural networks with time delays, Neurocomputing 363 (2019) 281-294.

[14] C. Wang, L. Xiong, J. Sun, W. Yao, Memristor-based neural networks with weight simultaneous perturbation training, Nonlinear Dynamics 95 (4) (2019) 2893-2906.

[15] L. Wang, M.-F. Ge, J. Hu, G. Zhang, Global stability and stabilization for inertial memristive neural networks with unbounded distributed delays, Nonlinear Dynamics 95 (2) (2019) 943-955.

[16] J. Qin, M. Li, Y. Shi, Q. Ma, W. X. Zheng, Optimal synchronization control of multiagent systems with input saturation via off-policy reinforcement learning, IEEE Transactions on Neural Networks and Learning Systems 30 (1) (2018) 85-96.

[17] L. Zhou, C. Wang, S. Du, L. Zhou, Cluster synchronization on multiple nonlinearly coupled dynamical subnetworks of complex networks with nonidentical nodes, IEEE Transactions on Neural Networks and Learning Systems 28 (3) (2016) 570-583. 
[29] N. Li, J. Cao, Lag synchronization of memristor-based coupled neural networks via omega-measure, IEEE Transactions on Neural Networks and Learning Systems 27 (3) (2015) 686-697. 
[30] C. Chen, L. Li, H. Peng, Y. Yang, Adaptive lag synchronization of memristive neural networks with mixed delays, IEEE Access 6 (2018) 40768-40777.

[31] Z. Guo, S. Gong, S. Wen, T. Huang, Event-based synchronization control for memristive neural networks with time-varying delay, IEEE Transactions on cybernetics 49 (9) (2018) 3268-3277.

[32] Y. Feng, X. Yang, Q. Song, J. Cao, Synchronization of memristive neural networks with mixed delays via quantized intermittent control, Applied Mathematics and Computation 339 (2018) 874-887.

[33] R. Wei, J. Cao, Fixed-time synchronization of quaternion-valued memristive neural networks with time delays, Neural Networks 113 (2019) 1-10.

[34] S. Ding, Z. Wang, Lag quasi-synchronization for memristive neural networks with switching jumps mismatch, Neural Computing and Applications 28 (12) (2017) 4011-4022.

[35] Y. Zhou, Z. Zeng, Event-triggered impulsive control on quasisynchronization of memristive neural networks with time-varying delays, Neural Networks 110 (2019) 55-65.

[36] Y. Xin, Y. Li, X. Huang, Z. Cheng, Quasi-synchronization of delayed chaotic memristive neural networks, IEEE Transactions on Cybernetics 49 (2) (2017) 712-718.

[37] Y. Fan, X. Huang, Y. Li, J. Xia, G. Chen, Aperiodically intermittent control for quasi-synchronization of delayed memristive neural networks: an interval matrix and matrix measure combined method, IEEE Transactions on Systems, Man, and Cybernetics: Systems (2018), doi:10.1109/TSMC.2018.2850157.

[38] X. Liu, T. Chen, J. Cao, W. Lu, Dissipativity and quasi-synchronization for neural networks with discontinuous activations and parameter mismatches, Neural Networks 24 (10) (2011) 1013-1021.

[39] R. Kumar, S. Das, Y. Cao, Effects of infinite occurrence of hybrid impulses with quasi-synchronization of parameter mismatched neural networks, Neural Networks 122 (2020) 106-116.

[40] L. Wang, Y. Shen, Q. Yin, G. Zhang, Adaptive synchronization of memristor-based neural networks with time-varying delays, IEEE Transactions on Neural Networks and Learning Systems 26 (9) (2014) 2033-2042.

[41] X. Liu, T. Chen, Synchronization of complex networks via aperiodically intermittent pinning control, IEEE Transactions on Automatic Control 60 (12) (2015) 3316-3321. 
[52] A. Halanay, A. Halanay, Differential equations: Stability, oscillations, time lags, Vol. 6, Academic press New York, 1966.

[53] X. Liu, T. Chen, Synchronization of linearly coupled networks with delays via aperiodically intermittent pinning control, IEEE Transactions on Neural Networks and Learning Systems 26 (10) (2015) 2396-2407. 
[54] L. Wang, Y. Shen, G. Zhang, Synchronization of a class of switched neural networks with time-varying delays via nonlinear feedback control, IEEE Transactions on Cybernetics 46 (10) (2015) 2300-2310.

[55] M. Jazayeri, J. A. Movshon, Optimal representation of sensory information by neural populations, Nature Neuroscience 9 (5) (2006) 690.

[56] M. J. Schnitzer, M. Meister, Multineuronal firing patterns in the signal from eye to brain, Neuron 37 (3) (2003) 499-511.

[57] Y. Kang, J. Qin, Q. Ma, H. Gao, W. X. Zheng, Cluster synchronization for interacting clusters of nonidentical nodes via intermittent pinning control, IEEE Transactions on Neural Networks and Learning Systems 29 (5) (2017) $1747-1759$.

[58] X. Yang, J. Lam, D. W. Ho, Z. Feng, Fixed-time synchronization of complex networks with impulsive effects via nonchattering control, IEEE Transactions on Automatic Control 62 (11) (2017) 5511-5521.

[59] X. Yang, Q. Song, J. Cao, J. Lu, Synchronization of coupled markovian reaction-diffusion neural networks with proportional delays via quantized control, IEEE Transactions on Neural Networks and Learning Systems 30 (3) (2018) 951-958. 